IFUP-TH/2013-17

\title{
REMARKS ON THE $U(1)$ AXIAL SYMMETRY AND THE CHIRAL TRANSITION IN QCD AT FINITE TEMPERATURE
}

\author{
Enrico Meggiolaro 1 * and Alessandro Mordà $2,3:$. \\ ${ }^{1}$ Dipartimento di Fisica, Università di Pisa, and INFN, Sezione di Pisa, \\ Largo Pontecorvo 3, I-56127 Pisa, Italy \\ ${ }^{2}$ CPPM, Aix-Marseille Université, CNRS/IN2P3, F-13288 Marseille, France \\ ${ }^{3}$ CPT, Aix-Marseille Université and Université du Sud Toulon-Var, \\ CNRS (UMR 7332), F-13288 Marseille, France
}

\begin{abstract}
We discuss the role of the $U(1)$ axial symmetry for the scalar and pseudoscalar meson mass spectrum of QCD at finite temperature, above the chiral transition at $T_{c}$, using a chiral effective Lagrangian model, which, in addition to the usual chiral condensate $\langle\bar{q} q\rangle$, also includes a (possible) genuine $U(1)_{A}$-breaking condensate that (possibly) survives across the chiral transition. The motivations for considering this Lagrangian (and a critical comparison with other effective Lagrangian models existing in the literature) are presented. A detailed comparison between the case $N_{f} \geq 3$ and the (remarkably different) case $N_{f}=2$ is performed. The results obtained in the case $N_{f}=2$ are also critically compared with the available lattice results.
\end{abstract}

PACS numbers: 11.10.Wx, 11.30.Rd, 12.38.Mh, 12.39.Fe

\footnotetext{
*E-mail: enrico.meggiolaro@df.unipi.it

${ }^{\dagger}$ E-mail: morda@cpt.univ-mrs.fr
} 


\section{Introduction}

It is well known that, at zero temperature, the $S U\left(N_{f}\right) \otimes S U\left(N_{f}\right)$ chiral symmetry of the QCD Lagrangian with $N_{f}$ massless quarks (the physically relevant cases being $N_{f}=2$ and $N_{f}=3$ ) is spontaneously broken down to the vectorial subgroup $S U\left(N_{f}\right)_{V}$ by the condensation of $q \bar{q}$ pairs, i.e., by the nonzero value of the vacuum expectation value $\langle\bar{q} q\rangle \equiv \sum_{l=1}^{N_{f}}\left\langle\bar{q}_{l} q_{l}\right\rangle$ (the so-called chiral condensate), and the $N_{f}^{2}-1 J^{P}=0^{-}$mesons are just the Goldstone bosons associated with this breaking (see, e.g., Ref. [1] and references therein). One expects that this scenario not only holds for massless quarks, but also continues for a small quark mass region, in which the Goldstone bosons become pseudoGoldstone bosons, with small (if compared with other hadrons) nonzero masses. The chiral condensate $\langle\bar{q} q\rangle$ is an order parameter for the chiral symmetry breaking: at high temperatures, the thermal energy breaks up the $q \bar{q}$ condensate, leading to the restoration of chiral symmetry for temperatures above the chiral phase transition temperature $T_{c}$, defined as the temperature at which the chiral condensate $\langle\bar{q} q\rangle$ goes to zero (in the chiral limit $m_{1}=\cdots=m_{N_{f}}=0$ ). From lattice determinations of $\langle\bar{q} q\rangle$, it is known (see, e.g., Refs. [2]) that this critical temperature is of the order $T_{c} \sim 150 \div 170 \mathrm{MeV}$ and practically equal to the deconfinement temperature $T_{d}$, separating the confined (or hadronic) phase at $T<T_{d}$, from the deconfined phase (also known as quark-gluon plasma) at $T>T_{d}$. But this is not the whole story, since, in addition to the $S U\left(N_{f}\right) \otimes S U\left(N_{f}\right)$ chiral symmetry, QCD with $N_{f}$ massless quarks also has a $U(1)$ axial symmetry (at least at the classical level) [3, 4]. This symmetry is broken by an anomaly at the quantum level, which in the Witten-Veneziano mechanism [5, 6] plays a fundamental role (via the so-called topological susceptibility) in explaining the large mass of the $\eta^{\prime}$ meson.

The role of the $U(1)$ axial symmetry for the finite temperature phase structure has been not well understood so far. One expects that at very high temperatures also the $U(1)$ axial symmetry will be (effectively) restored (since, at least for $T \gg T_{c}$, the density [in the partition function] of the instanton configurations, responsible for the $U(1)_{A}$ breaking, are strongly suppressed due to a Debye-type screening [7]); but it is still an open question of hadronic physics whether the fate of the $U(1)$ chiral symmetry of QCD has or has not something to do with the fate of the $S U\left(N_{f}\right) \otimes S U\left(N_{f}\right)$ chiral symmetry. This question is surely of phenomenological relevance since the particle mass spectrum above $T_{c}$ drastically depends on the presence or absence of the $U(1)$ axial symmetry. From the theoretical point of view, this question can be investigated by comparing the behavior at nonzero temperatures of the two-point correlation functions for the following $q \bar{q}$ meson channels 
(we consider for simplicity the case of $N_{f}=2$ light flavors) [8, 9]: the isoscalar $(I=0)$ scalar channel $\sigma$ (also known as $f_{0}$ in the modern language of hadron spectroscopy), interpolated by the operator $O_{\sigma}=\bar{q} q$; the isovector $(I=1)$ scalar channel $\vec{\delta}$ (also known as $\left.\vec{a}_{0}\right)$, interpolated by the operator $\vec{O}_{\delta}=\bar{q} \frac{\vec{\tau}}{2} q$; the isoscalar $(I=0)$ pseudoscalar channel $\eta$, interpolated by the operator $O_{\eta}=i \bar{q} \gamma_{5} q$; and the isovector $(I=1)$ pseudoscalar channel $\vec{\pi}$, interpolated by the operator $\vec{O}_{\pi}=i \bar{q} \gamma_{5} \frac{\vec{\tau}}{2} q$. Under $S U(2)_{A}$ transformations, $\sigma$ is mixed with $\vec{\pi} \mathrm{r}$;: thus, the restoration of this symmetry at $T_{c}$ requires identical correlators for these two channels, which implies, in particular, identical chiral susceptibilities, $\chi_{\sigma}=\chi_{\pi}$ $\left[\chi_{f} \equiv \int d^{4} x\left\langle T O_{f}(x) O_{f}^{\dagger}(0)\right\rangle\right]$, and identical (screening) masses, $M_{\sigma}=M_{\pi}$. Another $S U(2)$ chiral multiplet is $(\eta, \vec{\delta})$. On the contrary, under $U(1)_{A}$ transformations, $\vec{\delta}$ is mixed with $\vec{\pi}$, so, an effective restoration of the $U(1)$ axial symmetry should imply that these two channels become degenerate, with identical correlators and, therefore, with identical chiral susceptibilities, $\chi_{\delta}=\chi_{\pi}$, and identical (screening) masses, $M_{\delta}=M_{\pi}$. Another $U(1)$ chiral multiplet is $(\sigma, \eta)$. (Clearly, if both chiral symmetries are restored, then all $\sigma, \vec{\pi}$, $\eta$, and $\vec{\delta}$ correlators should become the same.)

In this paper, we shall analyze the scalar and pseudoscalar meson mass spectrum, above the chiral transition at $T_{c}$, using a chiral effective Lagrangian model (which was originally proposed in Refs. [10, 11, 12] and elaborated on in Refs. [13, 14, 15]), which, in addition to the usual chiral condensate $\langle\bar{q} q\rangle$, also includes a (possible) genuine $U(1)_{A^{-}}$ breaking condensate that (possibly) survives across the chiral transition at $T_{c}$, staying different from zero at $T>T_{c}$. The motivations for considering this Lagrangian (and a critical comparison with other effective Lagrangian models existing in the literature) are presented in Sec. 2. The results for the mesonic mass spectrum for $T>T_{c}$ are derived in Sec. 3, for the case $N_{f} \geq 3$, and in Sec. 4, for the case $N_{f}=2$. Finally, in Sec. 5, we shall summarize the results that we have obtained and we shall make some comments on (i) the remarkable difference between the case $N_{f} \geq 3$ and the case $N_{f}=2$ and (ii) the comparison between our results and the available lattice results for $N_{f}=2$.

\section{Chiral effective Lagrangians}

Chiral symmetry restoration at nonzero temperature is often studied in the framework of the following effective Lagrangian [16, 17, 18, 19] (which had been originally proposed to study the chiral dynamics at $T=0[20,21,22]$ ), written in terms of the (quark-bilinear) 
mesonic effective field $U_{i j} \sim \bar{q}_{j R} q_{i L}=\bar{q}_{j}\left(\frac{1+\gamma^{5}}{2}\right) q_{i}$ (up to a multiplicative constant) **

$$
\mathcal{L}_{1}\left(U, U^{\dagger}\right)=\mathcal{L}_{0}\left(U, U^{\dagger}\right)+\frac{B_{m}}{2 \sqrt{2}} \operatorname{Tr}\left[M U+M^{\dagger} U^{\dagger}\right]+\mathcal{L}_{I}\left(U, U^{\dagger}\right),
$$

where $\mathcal{L}_{0}\left(U, U^{\dagger}\right)$ describes a kind of linear sigma model:

$$
\begin{aligned}
\mathcal{L}_{0}\left(U, U^{\dagger}\right) & =\frac{1}{2} \operatorname{Tr}\left[\partial_{\mu} U \partial^{\mu} U^{\dagger}\right]-V_{0}\left(U, U^{\dagger}\right), \\
V_{0}\left(U, U^{\dagger}\right) & =\frac{1}{4} \lambda_{\pi}^{2} \operatorname{Tr}\left[\left(U U^{\dagger}-\rho_{\pi} \mathbf{I}\right)^{2}\right]+\frac{1}{4} \lambda_{\pi}^{\prime 2}\left[\operatorname{Tr}\left(U U^{\dagger}\right)\right]^{2} .
\end{aligned}
$$

I is the identity matrix, $M=\operatorname{diag}\left(m_{1}, \ldots, m_{N_{f}}\right)$ represents the quark mass matrix, which enters in the QCD Lagrangian as $\delta \mathcal{L}_{Q C D}^{(\text {mass })}=-\bar{q}_{R} M q_{L}-\bar{q}_{L} M^{\dagger} q_{R}$, while $\mathcal{L}_{I}\left(U, U^{\dagger}\right)$ is an interaction term of the form:

$$
\mathcal{L}_{I}\left(U, U^{\dagger}\right)=c_{I}\left[\operatorname{det} U+\operatorname{det} U^{\dagger}\right]
$$

Since under $U\left(N_{f}\right)_{L} \otimes U\left(N_{f}\right)_{R}$ chiral transformations the quark fields and the mesonic effective field $U$ transform as

$$
U\left(N_{f}\right)_{L} \otimes U\left(N_{f}\right)_{R}: \quad q_{L} \rightarrow V_{L} q_{L}, \quad q_{R} \rightarrow V_{R} q_{R} \Rightarrow U \rightarrow V_{L} U V_{R}^{\dagger}
$$

where $V_{L}$ and $V_{R}$ are arbitrary $N_{f} \times N_{f}$ unitary matrices, we have that $\mathcal{L}_{0}\left(U, U^{\dagger}\right)$ is invariant under the entire chiral group $U\left(N_{f}\right)_{L} \otimes U\left(N_{f}\right)_{R}$, while the interaction term (2.3) [and so the entire effective Lagrangian (2.1) in the chiral limit $M=0$ ] is invariant under $S U\left(N_{f}\right)_{L} \otimes S U\left(N_{f}\right)_{R} \otimes U(1)_{V}$ but not under a $U(1)$ axial transformation:

$$
U(1)_{A}: \quad q_{L} \rightarrow e^{-i \alpha} q_{L}, \quad q_{R} \rightarrow e^{i \alpha} q_{R} \Rightarrow U \rightarrow e^{-i 2 \alpha} U .
$$

It is often claimed (see, for example, Ref. [23] and references therein) that instanton processes, which are known to break the $U(1)_{A}$ symmetry by means of an effective $2 N_{f^{-}}$ quark vertex that is invariant under $S U\left(N_{f}\right)_{L} \otimes S U\left(N_{f}\right)_{R} \otimes U(1)_{V}$, but not under a $U(1)$ axial transformation, can be modelled using the interaction term (2.3).

However, as was noticed by Witten [24], Di Vecchia, and Veneziano [25], this type of anomalous term does not correctly reproduce the $\mathrm{U}(1)$ axial anomaly of the fundamental

*Throughout this paper, we use the following notations for the left-handed and right-handed quark fields: $q_{L, R} \equiv \frac{1}{2}\left(1 \pm \gamma_{5}\right) q$, with $\gamma_{5} \equiv-i \gamma^{0} \gamma^{1} \gamma^{2} \gamma^{3}$.

${ }^{\dagger}$ For the case of $N_{f}=2$ flavors, two other four-point couplings with the same property, i.e., invariant under $S U\left(N_{f}\right)_{L} \otimes S U\left(N_{f}\right)_{R} \otimes U(1)_{V}$ but not under $U(1)_{A}$, could be considered [16, 19]; however, these terms are not relevant for the type of analysis that we are going to perform in this paper. 
theory, i.e., of the QCD (and, moreover, it is inconsistent with the $1 / N_{c}$ expansion). In fact, one should require that, under a $U(1)$ axial transformation (2.5), the effective Lagrangian, in the chiral limit $M=0$, transforms as

$$
U(1)_{A}: \quad \mathcal{L}_{e f f}^{(M=0)}\left(U, U^{\dagger}, Q\right) \rightarrow \mathcal{L}_{e f f}^{(M=0)}\left(U, U^{\dagger}, Q\right)+\alpha 2 N_{f} Q
$$

where $Q(x)=\frac{g^{2}}{64 \pi^{2}} \varepsilon^{\mu \nu \rho \sigma} F_{\mu \nu}^{a}(x) F_{\rho \sigma}^{a}(x)$ is the topological charge density and $\mathcal{L}_{\text {eff }}$ also contains $Q$ as an auxiliary field. The correct effective Lagrangian, satisfying the transformation property (2.6), was derived in Refs. [24, 25, 26, 27, 28] and is given by

$$
\mathcal{L}_{2}\left(U, U^{\dagger}, Q\right)=\mathcal{L}_{0}\left(U, U^{\dagger}\right)+\frac{B_{m}}{2 \sqrt{2}} \operatorname{Tr}\left[M U+M^{\dagger} U^{\dagger}\right]+\frac{i}{2} Q \operatorname{Tr}\left[\log U-\log U^{\dagger}\right]+\frac{1}{2 A} Q^{2},
$$

where $A=-\left.i \int d^{4} x\langle T Q(x) Q(0)\rangle\right|_{Y M}$ is the so-called topological susceptibility in the pure Yang-Mills (YM) theory. After integrating out the variable $Q$ in the effective Lagrangian (2.7), we are left with

$$
\mathcal{L}_{2}\left(U, U^{\dagger}\right)=\mathcal{L}_{0}\left(U, U^{\dagger}\right)+\frac{B_{m}}{2 \sqrt{2}} \operatorname{Tr}\left[M U+M^{\dagger} U^{\dagger}\right]+\frac{1}{8} A\left\{\operatorname{Tr}\left[\log U-\log U^{\dagger}\right]\right\}^{2},
$$

to be compared with Eqs. (2.1)-(2.3).

For studying the phase structure of the theory at finite temperature, all the parameters appearing in the effective Lagrangian must be considered as functions of the physical temperature $T$. In particular, the parameter $\rho_{\pi}$, appearing in the first term of the potential $V_{0}\left(U, U^{\dagger}\right)$ in Eq. (2.2), is responsible for the behavior of the theory across the chiral phase transition at $T=T_{c}$. Let us consider, for a moment, only the linear sigma model $\mathcal{L}_{0}\left(U, U^{\dagger}\right)$, i.e., let us neglect both the anomalous symmetry-breaking term and the mass term in Eq. (2.8). If $\rho_{\pi}\left(T<T_{c}\right)>0$, then the value $\bar{U}$ for which the potential $V_{0}$ is minimum (that is, in a mean-field approach, the vacuum expectation value of the mesonic field $U$ ) is different from zero and can be chosen to be

$$
\left.\bar{U}\right|_{\rho_{\pi}>0}=v \mathbf{I}, \quad v \equiv \frac{F_{\pi}}{\sqrt{2}}=\sqrt{\frac{\rho_{\pi} \lambda_{\pi}^{2}}{\lambda_{\pi}^{2}+N_{f} \lambda_{\pi}^{\prime 2}}},
$$

which is invariant under the vectorial $U\left(N_{f}\right)_{V}$ subgroup; the chiral symmetry is thus spontaneously broken down to $U\left(N_{f}\right)_{V}$. Instead, if $\rho_{\pi}\left(T>T_{c}\right)<0$, we have that

$$
\left.\bar{U}\right|_{\rho_{\pi}<0}=0
$$


and the chiral symmetry is realized $\grave{a}$ la Wigner-Weyl. The critical temperature $T_{c}$ for the chiral phase transition is thus, in this case, simply the temperature at which the parameter $\rho_{\pi}$ vanishes: $\rho_{\pi}\left(T_{c}\right)=0$.

For $T>T_{c}$, where $\rho_{\pi}<0$ and $\bar{U}=0$, it is convenient to use for the matrix field $U$ the simple linear parametrization

$$
U_{i j}=a_{i j}+i b_{i j}
$$

where $a_{i j}$ and $b_{i j}$ are $2 N_{f}^{2}$ real fields, for which the vacuum expectation values vanish $\left(\bar{a}_{i j}=\right.$ $\bar{b}_{i j}=0$ ). Inserting Eq. (2.11) into Eq. (2.2), and putting $\rho_{\pi} \equiv-\frac{1}{2} B_{\pi}^{2}$, we find that, up to terms of second order in the fields, $\mathcal{L}_{0}=\frac{1}{2} \partial_{\mu} a_{i j} \partial^{\mu} a_{i j}+\frac{1}{2} \partial_{\mu} b_{i j} \partial^{\mu} b_{i j}-\frac{1}{4} \lambda_{\pi}^{2} B_{\pi}^{2}\left(a_{i j}^{2}+b_{i j}^{2}\right)+\ldots$, i.e., we have $2 N_{f}^{2}$ mesonic excitations with equal squared masses $M_{U}^{2}=\frac{1}{2} \lambda_{\pi}^{2} B_{\pi}^{2}$.

Instead, for $T<T_{c}$, where $\rho_{\pi}>0$ and $\bar{U}=\frac{F_{\pi}}{\sqrt{2}} \mathbf{I}$, it is more convenient to use for the matrix field $U$ the nonlinear parametrization (polar decomposition)

$$
U(x)=H(x) \Gamma(x)=\left(\frac{F_{\pi}}{\sqrt{2}} \mathbf{I}+\tilde{H}(x)\right) e^{i \frac{\sqrt{2}}{F_{\pi}} \Phi(x)}
$$

where $H=\frac{F_{\pi}}{\sqrt{2}} \mathbf{I}+\tilde{H}$ is an Hermitian $N_{f} \times N_{f}$ matrix, while $\Gamma=e^{i \frac{\sqrt{2}}{F_{\pi}} \Phi}$ is a unitary $N_{f} \times N_{f}$ matrix; i.e., $\tilde{H}(x)=\frac{1}{\sqrt{2}} \sum_{a} h_{a}(x) \tau^{a}+\frac{1}{\sqrt{N_{f}}} h_{0}(x) \mathbf{I}$ and $\Phi(x)=\frac{1}{\sqrt{2}} \sum_{a} \pi_{a}(x) \tau^{a}+\frac{1}{\sqrt{N_{f}}} S_{\pi}(x) \mathbf{I}$ are two Hermitian matrix fields, where $\tau^{a}\left(a=1, \ldots, N_{f}^{2}-1\right)$ are the generators of the $S U\left(N_{f}\right)$ algebra in the fundamental representation, with the normalization $\operatorname{Tr}\left(\tau^{a} \tau^{b}\right)=$ $2 \delta_{a b}$ (for $N_{f}=2$, they are the Pauli matrices, while for $N_{f}=3$, they are the Gell-Mann matrices), and $h_{a}, h_{0}$ are scalar mesonic fields, while $\pi_{a}, S_{\pi}$ are pseudoscalar mesonic fields, for which the vacuum expectation values vanish $\left(\bar{h}_{a}=\bar{h}_{0}=\bar{\pi}_{a}=\bar{S}_{\pi}=0\right)$. Inserting Eq. (2.12) into Eq. (2.2), and making use of Eq. (2.9), we find that the fields $\pi_{a}$ and $S_{\pi}$ are massless, and they are just the $N_{f}^{2}$ (pseudoscalar) Goldstone bosons generated by the spontaneous breaking of the chiral symmetry down to the $U\left(N_{f}\right)_{V}$ subgroup, while the (scalar) fields $h_{a}\left(a=1, \ldots, N_{f}^{2}-1\right)$ and $h_{0}$ have nonzero squared masses, respectively, given by $M_{a}^{2}=\lambda_{\pi}^{2} F_{\pi}^{2}$ and $M_{0}^{2}=\left(\lambda_{\pi}^{2}+N_{f} \lambda_{\pi}^{\prime 2}\right) F_{\pi}^{2} \oplus$

\footnotetext{
${ }^{\ddagger}$ If one is interested, e.g., at $T=0$, only in the lowest-energy effective states, i.e., only in the pseudoscalar mesonic excitations, one can formally decouple the massive scalar excitations $\tilde{H}$, by taking the limit $\lambda_{\pi}^{2} \rightarrow \infty$, which is a "static," i.e., infinite-mass, limit for $\tilde{H}$, and thus implies $\tilde{H} \rightarrow 0$. In this limit, the expression (2.12) for the mesonic field $U$ reduces to $U=\frac{F_{\pi}}{\sqrt{2}} e^{i \frac{\sqrt{2}}{F_{\pi}} \Phi}$, i.e., $U U^{\dagger}=\frac{F_{\pi}^{2}}{2} \mathbf{I}$, and the effective Lagrangian with this constraint becomes a nonlinear sigma model. We also observe that the quantity $F_{\pi}$, defined in Eq. (2.9) as $F_{\pi} \equiv \sqrt{2} v$, is just the usual pion decay constant, since the $S U\left(N_{f}\right)$ axial currents turn out to be, using Eq. (2.12), $A_{a}^{\mu}=\frac{i}{2} \operatorname{Tr}\left[T^{a}\left\{\partial^{\mu} U, U^{\dagger}\right\}\right]-\frac{i}{2} \operatorname{Tr}\left[T^{a}\left\{\partial^{\mu} U^{\dagger}, U\right\}\right]=-\sqrt{2} v \partial^{\mu} \pi_{a}+\cdots \equiv$ $-F_{\pi} \partial^{\mu} \pi_{a}+\ldots$
} 
If we now take into account the anomalous term in Eq. (2.8) (while keeping, for simplicity, the chiral limit $M=0$ ), it is easy to see that, for $T<T_{c}$, it modifies the result simply by adding a quadratic term in the pseudoscalar singlet field $S_{\pi}$,

$$
\mathcal{L}_{2}^{(M=0)}=\mathcal{L}_{0}-\frac{1}{2}\left(\frac{2 N_{f} A}{F_{\pi}^{2}}\right) S_{\pi}^{2},
$$

from which one derives the famous Witten-Veneziano formula for the singlet squared mass (in the chiral limit): $M_{S_{\pi}}^{2}=\frac{2 N_{f} A}{F_{\pi}^{2}}$. However, the anomalous term in Eq. (2.8) makes sense only in the low-temperature phase $\left(T<T_{c}\right)$, and it is singular for $T>T_{c}$, where the vacuum expectation value of the mesonic field $U$ vanishes. On the contrary, the interaction term (2.3) behaves well both in the low- and high-temperature phases.

\section{A. Effective Lagrangian with the inclusion of a $U(1)$ axial condensate}

The above-mentioned problems can be overcome by considering a modified effective Lagrangian (which was originally proposed in Refs. [10, 11, 12] and elaborated on in Refs. [13, 14, 15]), which generalizes the Lagrangian $\mathcal{L}_{2}$ written in Eq. (2.7), so that it correctly satisfies the transformation property (2.6) under the chiral group, but also includes an interaction term containing the determinant of the mesonic field $U$, of the kind of that in Eq. (2.3), assuming that there is a $U(1)_{A}$-breaking condensate that (possibly) survives across the chiral transition at $T_{c}$, staying different from zero up to a temperature $T_{U(1)}>T_{c}$. (Of course, it is also possible that $T_{U(1)} \rightarrow \infty$, as a limit case. Another possible limit case, i.e., $T_{U(1)}=T_{c}$, will be discussed in the concluding comments in Sec. 5.) The new $U(1)$ chiral condensate has the form $C_{U(1)}=\left\langle\mathcal{O}_{U(1)}\right\rangle$, where, for a theory with $N_{f}$ light quark flavors, $\mathcal{O}_{U(1)}$ is a $2 N_{f}$-quark local operator that has the chiral transformation properties of [4, 29, 30] $\mathcal{O}_{U(1)} \sim \operatorname{det}\left(\bar{q}_{s R} q_{t L}\right)+\operatorname{det}_{s t}\left(\bar{q}_{s L} q_{t R}\right)$, where $s, t=1, \ldots, N_{f}$ are flavor indices. The color indices (not explicitly indicated) are arranged in such a way that (i) $\mathcal{O}_{U(1)}$ is a color singlet, and (ii) $C_{U(1)}=\left\langle\mathcal{O}_{U(1)}\right\rangle$ is a genuine $2 N_{f}$-quark condensate, i.e., it has no disconnected part proportional to some power of the quark-antiquark chiral condensate $\langle\bar{q} q\rangle$; the explicit form of the condensate for the cases $N_{f}=2$ and $N_{f}=3$ is discussed in detail in the Appendix A of Ref. [15] (see also Refs. [12, 31]).

The modified effective Lagrangian is written in terms of the topological charge density $Q$, the mesonic field $U_{i j} \sim \bar{q}_{j R} q_{i L}$ (up to a multiplicative constant), and the new field variable $X \sim \operatorname{det}\left(\bar{q}_{s R} q_{t L}\right)$ (up to a multiplicative constant), associated with the $U(1)$ 
axial condensate [10, 11, 12],

$$
\begin{gathered}
\mathcal{L}\left(U, U^{\dagger}, X, X^{\dagger}, Q\right)=\frac{1}{2} \operatorname{Tr}\left[\partial_{\mu} U \partial^{\mu} U^{\dagger}\right]+\frac{1}{2} \partial_{\mu} X \partial^{\mu} X^{\dagger} \\
-V\left(U, U^{\dagger}, X, X^{\dagger}\right)+\frac{i}{2} \omega_{1} Q \operatorname{Tr}\left[\log U-\log U^{\dagger}\right] \\
+\frac{i}{2}\left(1-\omega_{1}\right) Q\left[\log X-\log X^{\dagger}\right]+\frac{1}{2 A} Q^{2},
\end{gathered}
$$

where the potential term $V\left(U, U^{\dagger}, X, X^{\dagger}\right)$ has the form

$$
\begin{aligned}
V\left(U, U^{\dagger}, X, X^{\dagger}\right) & =\frac{1}{4} \lambda_{\pi}^{2} \operatorname{Tr}\left[\left(U U^{\dagger}-\rho_{\pi} \mathbf{I}\right)^{2}\right]+\frac{1}{4} \lambda_{\pi}^{\prime 2}\left[\operatorname{Tr}\left(U U^{\dagger}\right)\right]^{2}+\frac{1}{4} \lambda_{X}^{2}\left[X X^{\dagger}-\rho_{X}\right]^{2} \\
& -\frac{B_{m}}{2 \sqrt{2}} \operatorname{Tr}\left[M U+M^{\dagger} U^{\dagger}\right]-\frac{c_{1}}{2 \sqrt{2}}\left[X^{\dagger} \operatorname{det} U+X \operatorname{det} U^{\dagger}\right] .
\end{aligned}
$$

Since under chiral $U\left(N_{f}\right)_{L} \otimes U\left(N_{f}\right)_{R}$ transformations [see Eq. (2.4)] the field $X$ transforms exactly as $\operatorname{det} U$,

$$
U\left(N_{f}\right)_{L} \otimes U\left(N_{f}\right)_{R}: \quad X \rightarrow \operatorname{det}\left(V_{L}\right) \operatorname{det}\left(V_{R}\right)^{*} X
$$

[i.e., $X$ is invariant under $S U\left(N_{f}\right)_{L} \otimes S U\left(N_{f}\right)_{R} \otimes U(1)_{V}$, while, under a $U(1)$ axial transformation (2.5), $\left.X \rightarrow e^{-i 2 N_{f} \alpha} X\right]$, we have that, in the chiral limit $M=0$, the effective Lagrangian (2.14) is invariant under $S U\left(N_{f}\right)_{L} \otimes S U\left(N_{f}\right)_{R} \otimes U(1)_{V}$, while under a $U(1)$ axial transformation, it correctly transforms as in Eq. (2.6).

After integrating out the variable $Q$ in the effective Lagrangian (2.14), we are left with

$$
\mathcal{L}\left(U, U^{\dagger}, X, X^{\dagger}\right)=\frac{1}{2} \operatorname{Tr}\left[\partial_{\mu} U \partial^{\mu} U^{\dagger}\right]+\frac{1}{2} \partial_{\mu} X \partial^{\mu} X^{\dagger}-\tilde{V}\left(U, U^{\dagger}, X, X^{\dagger}\right),
$$

where

$$
\begin{aligned}
\tilde{V}\left(U, U^{\dagger}, X, X^{\dagger}\right) & =V\left(U, U^{\dagger}, X, X^{\dagger}\right) \\
& -\frac{1}{8} A\left\{\omega_{1} \operatorname{Tr}\left[\log U-\log U^{\dagger}\right]+\left(1-\omega_{1}\right)\left[\log X-\log X^{\dagger}\right]\right\}^{2}
\end{aligned}
$$

As we have already said, all the parameters appearing in the effective Lagrangian must be considered as functions of the physical temperature $T$. In particular, the parameters $\rho_{\pi}$ and $\rho_{X}$ determine the expectation values $\langle U\rangle$ and $\langle X\rangle$, and so they are responsible for the behavior of the theory across the $S U\left(N_{f}\right) \otimes S U\left(N_{f}\right)$ and the $U(1)$ chiral phase transitions. We shall assume that the parameters $\rho_{\pi}$ and $\rho_{X}$, as functions of the temperature $T$, behave 


\begin{tabular}{|l|c|c|}
\hline$T<T_{\rho_{\pi}}$ & $T_{\rho_{\pi}}<T<T_{U(1)}$ & $T>T_{U(1)}$ \\
\hline$\rho_{\pi}>0$ & $\rho_{\pi}<0$ & $\rho_{\pi}<0$ \\
\hline$\rho_{X}>0$ & $\rho_{X}>0$ & $\rho_{X}<0$ \\
\hline
\end{tabular}

Table 1: Dependence of the parameters $\rho_{\pi}$ and $\rho_{X}$ on the temperature $T$.

as reported in Table 1: $T_{\rho_{\pi}}$ is thus the temperature at which the parameter $\rho_{\pi}$ vanishes, while $T_{U(1)}>T_{\rho_{\pi}}$ is the temperature at which the parameter $\rho_{X}$ vanishes (with, as we have said above, $T_{U(1)} \rightarrow \infty$, i.e., $\rho_{X}>0 \forall T$, as a possible limit case). We shall see in the next section that, in the case $N_{f} \geq 3$, one has $T_{c}=T_{\rho_{\pi}}$ (exactly as in the case of the linear sigma model $\mathcal{L}_{0}$ discussed above), while, as we shall see in Sec. 4 , the situation in which $N_{f}=2$ is more complicated, being $T_{\rho_{\pi}}<T_{c}<T_{U(1)}$ in that case (unless $T_{\rho_{\pi}}=T_{c}=T_{U(1)}$; this limit case will be discussed in the concluding comments in Sec. 5).

Concerning the parameter $\omega_{1}$, in order to avoid a singular behavior of the anomalous term in Eq. (2.18) above the chiral transition temperature $T_{c}$, where the vacuum expectation value of the mesonic field $U$ vanishes (in the chiral limit $M=0$ ), we shall assume that $\omega_{1}\left(T \geq T_{c}\right)=0$.

Finally, let us observe that the interaction term between the $U$ and $X$ fields in Eq. (2.15), i.e.,

$$
\mathcal{L}_{i n t}=\frac{c_{1}}{2 \sqrt{2}}\left[X^{\dagger} \operatorname{det} U+X \operatorname{det} U^{\dagger}\right],
$$

is very similar to the interaction term (2.3) that we have discussed above for the effective Lagrangian $\mathcal{L}_{1}$. However, the term (2.19) is not anomalous, being invariant under the chiral group $U\left(N_{f}\right)_{L} \otimes U\left(N_{f}\right)_{R}$, by virtue of Eqs. (2.4) and (2.16). Nevertheless, if the field $X$ has a (real) nonzero vacuum expectation value $\bar{X}$ [the $U(1)$ axial condensate], then we can write

$$
X=\left(\bar{X}+h_{X}\right) e^{i \frac{S_{X}}{X}} \quad\left(\text { with }: \bar{h}_{X}=\bar{S}_{X}=0\right),
$$

and, after susbstituting this in Eq. (2.19) and expanding in powers of the excitations $h_{X}$ and $S_{X}$, one recovers, at the leading order, an interaction term of the form (2.3):

$$
\mathcal{L}_{i n t}=c_{I}\left[\operatorname{det} U+\operatorname{det} U^{\dagger}\right]+\ldots, \quad \text { with }: \quad c_{I}=\frac{c_{1} \bar{X}}{2 \sqrt{2}} .
$$

In the rest of this paper we shall analyze in detail the effects of assuming a nonzero value of the $U(1)$ axial condensate $\bar{X}$ on the scalar and pseudoscalar meson mass spectrum 
above the chiral transition temperature $\left(T>T_{c}\right.$ ), both for the case $N_{f} \geq 3$ (Sec. 3) and for the case $N_{f}=2$ (Sec. 4 ).

\section{Mass spectrum for $T>T_{c}$ in the case $N_{f} \geq 3$}

The results for the scalar and pseudoscalar meson mass spectrum for $T>T_{c}$ in the case $N_{f} \geq 3$ were rapidly sketched in Ref. [10] and, in this section, we shall rederive them in a more detailed and accurate way in order to allow for a more clear comparison with the novel results that we shall obtain in the next section for the case $N_{f}=2$.

Let us suppose to be in the range of temperatures $T_{\rho_{\pi}}<T<T_{U(1)}$, where, according to Table 1,

$$
\rho_{\pi} \equiv-\frac{1}{2} B_{\pi}^{2}<0, \quad \rho_{X} \equiv \frac{1}{2} F_{X}^{2}>0 .
$$

Since we expect that, due to the sign of the parameter $\rho_{X}$ in the potential (2.15), the $U(1)$ axial symmetry is broken by a nonzero vacuum expectation value of the field $X$ (at least for $\lambda_{X}^{2} \rightarrow \infty$ we should have $\bar{X}^{\dagger} \bar{X} \rightarrow \frac{1}{2} F_{X}^{2}$ ), we shall use for the field $U$ the linear parametrization (2.11), while for the field $X$, we shall use a nonlinear parametrization, similar to the polar decomposition in Eq. (2.12),

$$
U_{i j}=a_{i j}+i b_{i j}, \quad X=\alpha e^{i \beta}=\left(\bar{\alpha}+h_{X}\right) e^{i\left(\bar{\beta}+\frac{S_{X}}{\bar{\alpha}}\right)},
$$

where $\bar{X}=\bar{\alpha} e^{i \bar{\beta}}$ (with $\bar{\alpha} \neq 0$ ) is the vacuum expectation value of $X$ and $a_{i j}, b_{i j}, h_{X}$, and $S_{X}$ are real fields. Inserting Eq. (3.2) into the expressions (2.15) and (2.18), we find the expressions for the potential with and without the anomalous term (with $\omega_{1}=0$ ),

$$
\tilde{V}=V-\frac{1}{8} A\left[\log X-\log X^{\dagger}\right]^{2}=V+\frac{1}{2} A \beta^{2}
$$

and

$$
\begin{aligned}
V= & \frac{N_{f}}{16} \lambda_{\pi}^{2} B_{\pi}^{4}+\frac{1}{4} \lambda_{\pi}^{2} \operatorname{Tr}\left[\left(U U^{\dagger}\right)\left(U U^{\dagger}\right)\right]+\frac{1}{4} \lambda_{\pi}^{\prime 2}\left[\operatorname{Tr}\left(U U^{\dagger}\right)\right]^{2}+\frac{1}{4} \lambda_{X}^{2}\left(\alpha^{2}-\frac{1}{2} F_{X}^{2}\right)^{2} \\
& +\frac{1}{4} \lambda_{\pi}^{2} B_{\pi}^{2}\left(a_{i j}^{2}+b_{i j}^{2}\right)-\frac{B_{m}}{\sqrt{2}}\left(m_{i j} a_{j i}-n_{i j} b_{j i}\right) \\
& -\frac{c_{1}}{2 \sqrt{2}}\left[\alpha \cos \beta\left(\operatorname{det} U+\operatorname{det} U^{\dagger}\right)+i \alpha \sin \beta\left(\operatorname{det} U-\operatorname{det} U^{\dagger}\right)\right],
\end{aligned}
$$

where we have assumed the most general (complex) mass matrix $M_{i j}=m_{i j}+i n_{i j}$, with $m_{i j}$ and $n_{i j}$ real. Let us first look for the equations for a stationary point $(S)$ of the 
nonanomalous potential $V$, indicating with $\bar{U}$ and $\bar{X}$ the values of the fields $U$ and $X$ in this point:

$$
\begin{gathered}
\left.\frac{\partial V}{\partial a_{i j}}\right|_{S}=\frac{1}{2} \lambda_{\pi}^{2} B_{\pi}^{2} \bar{a}_{i j}-\frac{B_{m}}{\sqrt{2}} m_{j i}+\cdots=0 \\
\left.\frac{\partial V}{\partial b_{i j}}\right|_{S}=\frac{1}{2} \lambda_{\pi}^{2} B_{\pi}^{2} \bar{b}_{i j}+\frac{B_{m}}{\sqrt{2}} n_{j i}+\cdots=0 \\
\left.\frac{\partial V}{\partial \alpha}\right|_{S}=\lambda_{X}^{2}\left(\bar{\alpha}^{2}-\frac{F_{X}^{2}}{2}\right) \bar{\alpha}-\frac{c_{1}}{2 \sqrt{2}}\left[\cos \bar{\beta}\left(\operatorname{det} \bar{U}+\operatorname{det} \bar{U}^{\dagger}\right)\right. \\
\left.+i \sin \bar{\beta}\left(\operatorname{det} \bar{U}^{\dagger}-\operatorname{det} \bar{U}\right)\right]=0, \\
\left.\frac{\partial V}{\partial \beta}\right|_{S}=\frac{c_{1}}{2 \sqrt{2}} \bar{\alpha}\left[\sin \bar{\beta}\left(\operatorname{det} \bar{U}+\operatorname{det} \bar{U}^{\dagger}\right)-i \cos \bar{\beta}\left(\operatorname{det} \bar{U}^{\dagger}-\operatorname{det} \bar{U}\right)\right]=0 .
\end{gathered}
$$

From the first two equations, where we have omitted terms that, for $N_{f} \geq 3$, are of order 2 or higher in the fields $a$ and $b$, we find that, at the leading order in $M$,

$$
\bar{U}=\frac{2 B_{m}}{\sqrt{2} \lambda_{\pi}^{2} B_{\pi}^{2}} M^{\dagger}+\ldots
$$

Let us now consider the second derivatives of the potential $V$ with respect to the fields, calculated at the stationary point $S$ :

$$
\begin{gathered}
\left.\frac{\partial^{2} V}{\partial a_{l m} \partial a_{i j}}\right|_{S}=\frac{1}{2} \lambda_{\pi}^{2} B_{\pi}^{2} \delta_{i l} \delta_{j m}+\ldots, \\
\left.\frac{\partial^{2} V}{\partial b_{l m} \partial b_{i j}}\right|_{S}=\frac{1}{2} \lambda_{\pi}^{2} B_{\pi}^{2} \delta_{i l} \delta_{j m}+\ldots \\
\left.\frac{\partial^{2} V}{\partial \alpha^{2}}\right|_{S}=\lambda_{X}^{2}\left(3 \bar{\alpha}^{2}-\frac{F_{X}^{2}}{2}\right), \\
\left.\frac{\partial^{2} V}{\partial \beta^{2}}\right|_{S}=\frac{c_{1}}{2 \sqrt{2}} \bar{\alpha}\left[\cos \bar{\beta}\left(\operatorname{det} \bar{U}+\operatorname{det} \bar{U}^{\dagger}\right)+i \sin \bar{\beta}\left(\operatorname{det} \bar{U}^{\dagger}-\operatorname{det} \bar{U}\right)\right] \\
\left.\frac{\partial^{2} V}{\partial \alpha \partial \beta}\right|_{S}=\frac{c_{1}}{2 \sqrt{2}}\left[\sin \bar{\beta}\left(\operatorname{det} \bar{U}+\operatorname{det} \bar{U}^{\dagger}\right)-i \cos \bar{\beta}\left(\operatorname{det} \bar{U}^{\dagger}-\operatorname{det} \bar{U}\right)\right] .
\end{gathered}
$$

The first two equations are given at the leading order in the quark masses, and all the second derivatives, which are not shown in Eq. (3.7), are of order $\mathcal{O}(m)$ or higher in the quark masses. From the third equation of Eqs. (3.7), it is clear that the stationary point can be a minimum of the potential only for $\bar{\alpha} \neq 0$. If we now take for $M$ the physical real diagonal matrix $M=\operatorname{diag}\left(m_{1}, \ldots, m_{N_{f}}\right)$, we have that $M=M^{\dagger}$ and therefore, by virtue of the result (3.6), also $\bar{U}=\bar{U}^{\dagger}$. Indeed, this is a more general result, not directly related to the particular solution (3.6) (which, as we shall see in the next section, is valid 
for $N_{f} \geq 3$, but not for $N_{f}=2$ ), being due, when $M$ is a real diagonal matrix (or, more generally, when $M$ is Hermitian), to the invariance of the theory under parity $(P)$ transformations [i.e., being $U_{i j} \sim \bar{q}_{j R} q_{i L}$ and $X \sim \operatorname{det}\left(\bar{q}_{s R} q_{t L}\right), U\left(x^{0}, \vec{x}\right) \stackrel{P}{\rightarrow} U^{\dagger}\left(x^{0},-\vec{x}\right)$, $\left.X\left(x^{0}, \vec{x}\right) \stackrel{P}{\rightarrow} X^{\dagger}\left(x^{0},-\vec{x}\right)\right]$, which requires that $\bar{U}=\bar{U}^{\dagger}$ and $\bar{X}=\bar{X}^{\dagger}$. From the last of Eqs. (3.5), we thus find that $\sin \bar{\beta}=0$, i.e., $\bar{\beta}=0, \pi$, which also implies that $\left.\frac{\partial^{2} V}{\partial \alpha \partial \beta}\right|_{S}=0$. Moreover, from the fourth Eq. (3.7), using the result (3.6), it is clear that, for the stationary point $S$ to be a minimum, we must require, assuming $c_{1}>0$ and $B_{m}>0$, that also $\bar{\alpha} \cos \bar{\beta}>0$; so, finally, we can take $\bar{\alpha}>0$ and $\bar{\beta}=0$. We can then determine $\bar{\alpha}$ using the third equation in Eqs. (3.5) and so find

$$
\bar{\alpha}=\frac{F_{X}}{\sqrt{2}}+\frac{c_{1}}{\sqrt{2} \lambda_{X}^{2} F_{X}^{2}}\left(\frac{2 B_{m}}{\sqrt{2} \lambda_{\pi}^{2} B_{\pi}^{2}}\right)^{N_{f}} \operatorname{det} M+\ldots,
$$

which gives $\left.\frac{\partial^{2} V}{\partial \alpha^{2}}\right|_{S}=\lambda_{X}^{2} F_{X}^{2}+\mathcal{O}(\operatorname{det} M)$.

If we now consider the full potential $\tilde{V}$, with the inclusion of the anomalous term, see Eq. (3.3), it is trivial to see that the solution that we have found for the minimum of $V$, given by Eqs. (3.6) and (3.8) with $\bar{\beta}=0$, is also a minimum for the potential $\tilde{V}$, the only modification being in the second derivative of the potential with respect to $\beta$, which is now given by [see the fourth equation in Eqs. (3.7)] $]\left.\frac{\partial^{2} \tilde{V}}{\partial \beta^{2}}\right|_{S}=\left.\frac{\partial^{2} V}{\partial \beta^{2}}\right|_{S}+A=A+\mathcal{O}(\operatorname{det} M)$.

In particular, in the chiral limit $M=0$, we find that $\bar{U}=0$ and $\bar{X}=\bar{\alpha}=\frac{F_{X}}{\sqrt{2}}$, which means that, in this range of temperatures $T_{\rho_{\pi}}<T<T_{U(1)}$, the $S U\left(N_{f}\right)_{L} \otimes S U\left(N_{f}\right)_{R}$ chiral symmetry is restored so that we can say that (at least for $N_{f} \geq 3$ ) $T_{c} \equiv T_{\rho_{\pi}}$, while the $U(1)$ axial symmetry is broken by the $U(1)$ axial condensate $\bar{X}$. Concerning the mass spectrum of the effective Lagrangian, we have $2 N_{f}^{2}$ degenerate scalar and pseudoscalar mesonic excitations, described by the fields $a_{i j}$ and $b_{i j}$, plus a scalar singlet field $h_{X}=\alpha-\bar{\alpha}$ and a pseudoscalar singlet field $S_{X}=\bar{\alpha} \beta$ [see Eq. (3.2)], with squared masses given by

$$
M_{U}^{2}=\frac{1}{2} \lambda_{\pi}^{2} B_{\pi}^{2}, \quad M_{h_{X}}^{2}=\lambda_{X}^{2} F_{X}^{2}, \quad M_{S_{X}}^{2}=\frac{A}{\bar{\alpha}^{2}}=\frac{2 A}{F_{X}^{2}} .
$$

While the mesonic excitations described by the field $U$ are of the usual $q \bar{q}$ type, the scalar singlet field $h_{X}$ and the pseudoscalar singlet field $S_{X}$ describe instead two exotic, $2 N_{f^{-}}$ quark excitations of the form $h_{X} \sim \operatorname{det}\left(\bar{q}_{s L} q_{t R}\right)+\operatorname{det}\left(\bar{q}_{s R} q_{t L}\right)$ and $S_{X} \sim i\left[\operatorname{det}\left(\bar{q}_{s L} q_{t R}\right)-\right.$ $\left.\operatorname{det}\left(\bar{q}_{s R} q_{t L}\right)\right]$. In particular, the physical interpretation of the pseudoscalar singlet excitation $S_{X}$ is rather obvious, and it was already discussed in Ref. [10]: it is nothing but the would-be Goldstone particle coming from the breaking of the $U(1)$ axial symmetry. 
In fact, neglecting the anomaly, it has zero mass in the chiral limit of zero quark masses. Yet, considering the anomaly, it acquires a topological squared mass proportional to the topological susceptibility $A$ of the pure YM theory, as required by the Witten-Veneziano mechanism [5, 6].

\section{Mass spectrum for $T>T_{c}$ in the case $N_{f}=2$}

In this section we shall derive the results for the scalar and pseudoscalar mesonic mass spectrum for $T>T_{c}$ in the case $N_{f}=2$, with a quark mass matrix given by $M=$ $\operatorname{diag}\left(m_{u}, m_{d}\right)$.

As in the previous section, we start considering the range of temperatures $T_{\rho_{\pi}}<T<$ $T_{U(1)}$, with the parameters $\rho_{\pi}$ and $\rho_{X}$ given by Eq. (3.1) (see also Table 1). We shall use for the field $U$ a more convenient variant of the linear parametrization (2.11), while for the field $X$, we shall use the usual nonlinear parametrization given in Eq. (3.2) **

$$
U=\frac{1}{\sqrt{2}}[(\sigma+i \eta) \mathbf{I}+(\vec{\delta}+i \vec{\pi}) \cdot \vec{\tau}], \quad X=\alpha e^{i \beta}=\left(\bar{\alpha}+h_{X}\right) e^{i \frac{S_{X}}{\bar{\alpha}}},
$$

where $\tau^{a}(a=1,2,3)$ are the three Pauli matrices [with the usual normalization $\operatorname{Tr}\left(\tau^{a} \tau^{b}\right)=$ $\left.2 \delta_{a b}\right]$ and the multiplicative factor $\frac{1}{\sqrt{2}}$ guarantees the correct normalization of the kinetic term in the effective Lagrangian. The fields $\sigma, \eta, \vec{\delta}$, and $\vec{\pi}$ describe, respectively, the isoscalar $(I=0)$ scalar $q \bar{q}$ mesonic excitation $\sigma$ (also known as $f_{0}$ in the modern language of hadron spectroscopy), the isoscalar $(I=0)$ pseudoscalar $q \bar{q}$ mesonic excitation $\eta$, the isovector $(I=1)$ scalar $q \bar{q}$ mesonic excitation $\vec{\delta}$ (also known as $\vec{a}_{0}$ ), and the isovector $(I=1)$ pseudoscalar $q \bar{q}$ mesonic excitation $\vec{\pi}$.

Inserting Eq. (4.1) into the expressions (2.15) and (2.18), we find the following expression for the potential $V$, without the anomalous term,

$$
\begin{aligned}
V= & \frac{1}{8} \lambda_{\pi}^{2} B_{\pi}^{4}+\frac{1}{8} \Lambda_{\pi}^{2}\left(\sigma^{2}+\eta^{2}+\vec{\pi}^{2}+\vec{\delta}^{2}\right)^{2}+\frac{1}{2} \lambda_{\pi}^{2}\left(\sigma^{2} \vec{\delta}^{2}+2 \sigma \eta \vec{\delta} \cdot \vec{\pi}+\eta^{2} \vec{\pi}^{2}\right) \\
& +\frac{1}{2} \lambda_{\pi}^{2}\left[\vec{\pi}^{2} \vec{\delta}^{2}-(\vec{\pi} \cdot \vec{\delta})^{2}\right]+\frac{1}{4} \lambda_{\pi}^{2} B_{\pi}^{2}\left[\sigma^{2}+\eta^{2}+\vec{\delta}^{2}+\vec{\pi}^{2}\right]+\frac{1}{4} \lambda_{X}^{2}\left(\alpha^{2}-\frac{F_{X}^{2}}{2}\right)^{2} \\
& -\frac{B_{m}}{2}\left[\left(m_{u}+m_{d}\right) \sigma+\left(m_{u}-m_{d}\right) \delta_{3}\right] \\
& -\frac{c_{1}}{2 \sqrt{2}}\left[\alpha \cos \beta\left(\sigma^{2}-\eta^{2}-\vec{\delta}^{2}+\vec{\pi}^{2}\right)+2 \alpha \sin \beta(\sigma \eta-\vec{\delta} \cdot \vec{\pi})\right],
\end{aligned}
$$

${ }^{*}$ Here, we immediately put $\bar{\beta}=0$, since, as one can easily see, the arguments leading to $\bar{\beta}=0$, which we have given in the previous section, are valid also for $N_{f}=2$. 
where

$$
\Lambda_{\pi}^{2} \equiv \lambda_{\pi}^{2}+2 \lambda_{\pi}^{\prime 2}
$$

while the full potential $\tilde{V}$, including also the anomalous term (with $\omega_{1}=0$ ), is still given by Eq. (3.3), i.e., $\tilde{V}=V+\frac{1}{2} A \beta^{2}$.

When looking for the equations for a stationary point $(S)$ of the potential $\tilde{V}$, indicating as usual with $\bar{U}$ and $\bar{X}$ the values of the fields $U$ and $X$ in this point, we can immediately make use, with $M$ being a real diagonal (and, therefore, Hermitian) matrix, of the invariance of the theory under parity $(P)$ transformations (as already observed in the previous section), which requires that $\bar{U}=\bar{U}^{\dagger}$ and $\bar{X}=\bar{X}^{\dagger}$. That is to say, using the parametrization (4.1), $\bar{\eta}=\bar{\pi}_{a}=\bar{\beta}=0$. This automatically guarantees the vanishing of the first derivatives of the potential $\tilde{V}$ with respect to the pseudoscalar fields at the stationary point $S$, i.e., $\left.\frac{\partial \tilde{V}}{\partial \eta}\right|_{S}=\left.\frac{\partial \tilde{V}}{\partial \pi_{a}}\right|_{S}=\left.\frac{\partial \tilde{V}}{\partial \beta}\right|_{S}=0$, as one can easily verify using Eqs. (4.2) and (3.3).

Moreover, the vanishing, at the stationary point $(S)$, of the derivatives of Eq. (4.2) with respect to the fields $\delta_{a}(a=1,2,3)$, gives the following three equations:

$$
\left.\frac{\partial \tilde{V}}{\partial \delta_{a}}\right|_{S}=\frac{1}{2}\left[\Lambda_{\pi}^{2}\left(\bar{\sigma}^{2}+\overline{\bar{\delta}}^{2}\right)+2 \lambda_{\pi}^{2} \bar{\sigma}^{2}+\left(\lambda_{\pi}^{2} B_{\pi}^{2}+\sqrt{2} c_{1} \bar{\alpha}\right)\right] \bar{\delta}_{a}-\frac{1}{2} B_{m}\left(m_{u}-m_{d}\right) \delta_{a 3}=0 .
$$

For $a=1$ and $a=2$, using the fact that $c_{1}>0$ and $\bar{\alpha}>0$ (or, more generally, $c_{1} \bar{\alpha}>0$; see the discussion in the previous section, which can be easily extended also to the case $N_{f}=2$ considered here), one immediately finds the solution $\bar{\delta}_{1}=\bar{\delta}_{2}=0$. Let us also observe that, in the chiral limit $m_{u}=m_{d}=0$, or, more generally, in the limit of equal quark masses $m_{u}=m_{d}$, one also has $\bar{\delta}_{3}=0$ so that $\bar{U}=\frac{\bar{\sigma}}{\sqrt{2}} \mathbf{I}$, which is invariant under the $S U(2)_{V}$ (isospin) symmetry, as it must be.

So, finally, we are left with the following three equations for the values $\bar{\alpha}, \bar{\sigma}$ and $\bar{\delta} \equiv \bar{\delta}_{3}$ :

$$
\begin{aligned}
& \left.\frac{\partial \tilde{V}}{\partial \sigma}\right|_{S}=\frac{1}{2}\left[\Lambda_{\pi}^{2}\left(\bar{\sigma}^{2}+\bar{\delta}^{2}\right)+2 \lambda_{\pi}^{2} \bar{\delta}^{2}+\left(\lambda_{\pi}^{2} B_{\pi}^{2}-\sqrt{2} c_{1} \bar{\alpha}\right)\right] \bar{\sigma}-\frac{1}{2} B_{m}\left(m_{u}+m_{d}\right)=0 \\
& \left.\frac{\partial \tilde{V}}{\partial \delta_{3}}\right|_{S}=\frac{1}{2}\left[\Lambda_{\pi}^{2}\left(\bar{\sigma}^{2}+\bar{\delta}^{2}\right)+2 \lambda_{\pi}^{2} \bar{\sigma}^{2}+\left(\lambda_{\pi}^{2} B_{\pi}^{2}+\sqrt{2} c_{1} \bar{\alpha}\right)\right] \bar{\delta}-\frac{1}{2} B_{m}\left(m_{u}-m_{d}\right)=0 \\
& \left.\frac{\partial \tilde{V}}{\partial \alpha}\right|_{S}=\lambda_{X}^{2}\left(\bar{\alpha}^{2}-\frac{F_{X}^{2}}{2}\right) \bar{\alpha}-\frac{c_{1}}{2 \sqrt{2}}\left(\bar{\sigma}^{2}-\bar{\delta}^{2}\right)=0 .
\end{aligned}
$$

It is easy to see that Eqs. (4.5) admit the following solution (at the first nontrivial order 
in the quark masses $m_{u}$ and $m_{d}$ ),

$$
\begin{aligned}
\bar{\sigma} & =\frac{B_{m}}{\lambda_{\pi}^{2} B_{\pi}^{2}-c_{1} F_{X}}\left(m_{u}+m_{d}\right)+\ldots, \\
\bar{\delta} & =\frac{B_{m}}{\lambda_{\pi}^{2} B_{\pi}^{2}+c_{1} F_{X}}\left(m_{u}-m_{d}\right)+\ldots, \\
\bar{\alpha} & =\frac{F_{X}}{\sqrt{2}}+\frac{\sqrt{2} c_{1}^{2} \lambda_{\pi}^{2} B_{\pi}^{2}}{\lambda_{X}^{2} F_{X}\left(\lambda_{\pi}^{4} B_{\pi}^{4}-c_{1}^{2} F_{X}^{2}\right)^{2}} B_{m}^{2}\left(m_{u}^{2}+m_{d}^{2}\right) \\
& +\frac{\sqrt{2} c_{1}\left(\lambda_{\pi}^{4} B_{\pi}^{4}+c_{1}^{2} F_{X}^{2}\right)}{\lambda_{X}^{2} F_{X}^{2}\left(\lambda_{\pi}^{4} B_{\pi}^{4}-c_{1}^{2} F_{X}^{2}\right)^{2}} B_{m}^{2} m_{u} m_{d}+\ldots,
\end{aligned}
$$

which, in the chiral limit $m_{u}=m_{d}=0$, reduces to

$$
\bar{\sigma}=\bar{\delta}=0, \quad \bar{\alpha}=\frac{F_{X}}{\sqrt{2}}, \quad \text { i.e. : } \quad \bar{U}=0, \quad \bar{X}=\bar{\alpha}=\frac{F_{X}}{\sqrt{2}},
$$

signalling that the $S U\left(N_{f}\right)_{L} \otimes S U\left(N_{f}\right)_{R}$ chiral symmetry is restored, while the $U(1)$ axial symmetry is broken by the $U(1)$ axial condensate $\bar{X}$.

To see if this stationary point is a minimum of the potential (and, eventually, in order to derive the mass spectrum of the effective Lagrangian), we must study the matrix of the second derivatives (Hessian) of the potential $\tilde{V}$ with respect to the fields at the stationary point $S$. By virtue of the parity invariance of the theory, one immediately has that the mixed second derivatives of $\tilde{V}$ with respect to a scalar field and a pseudoscalar field vanish at the stationary point $S$, as one can easily verify using Eqs. (4.2) and (3.3). In other words, the scalar sector $\left(h_{X}, \sigma, \vec{\delta}\right)$ and the pseudoscalar sector $\left(S_{X}, \eta, \vec{\pi}\right)$ are decoupled in the matrix of the second derivatives of $\tilde{V}$ at the stationary point $S$, and, therefore, they can be studied separately.

\section{A. Scalar sector}

From Eqs. (4.2) and (3.3), it comes out that the Hessian matrix (evaluated at the stationary point $S$ ) is already diagonal with respect to the fields $\delta_{1}$ and $\delta_{2}$, with a common value of the squared masses given by

$$
M_{\delta_{1,2}}^{2}=\frac{1}{2}\left(\lambda_{\pi}^{2} B_{\pi}^{2}+c_{1} \sqrt{2} \bar{\alpha}\right)+\frac{1}{2} \Lambda_{\pi}^{2}\left(\bar{\sigma}^{2}+\bar{\delta}^{2}\right)+\lambda_{\pi}^{2} \bar{\sigma}^{2}
$$

The Hessian of the remaining scalar fields $\left(h_{X}, \sigma, \delta_{3}\right)$ turns out to be

$$
\mathcal{H}_{(S)}=\left(\begin{array}{ccc}
\lambda_{X}^{2}\left(3 \bar{\alpha}^{2}-\frac{F_{X}^{2}}{2}\right) & -\frac{c_{1}}{\sqrt{2}} \bar{\sigma} & \frac{c_{1}}{\sqrt{2}} \bar{\delta} \\
-\frac{c_{1}}{\sqrt{2}} \bar{\sigma} & \frac{1}{2}\left(\lambda_{\pi}^{2} B_{\pi}^{2}-c_{1} \sqrt{2} \bar{\alpha}\right)+\Delta_{\sigma} & \left(\Lambda_{\pi}^{2}+2 \lambda_{\pi}^{2}\right) \bar{\sigma} \bar{\delta} \\
\frac{c_{1}}{\sqrt{2}} \bar{\delta} & \left(\Lambda_{\pi}^{2}+2 \lambda_{\pi}^{2}\right) \bar{\sigma} \bar{\delta} & \frac{1}{2}\left(\lambda_{\pi}^{2} B_{\pi}^{2}+c_{1} \sqrt{2} \bar{\alpha}\right)+\Delta_{\delta}
\end{array}\right)
$$


where $\Delta_{\sigma} \equiv \frac{3}{2} \Lambda_{\pi}^{2} \bar{\sigma}^{2}+\frac{1}{2}\left(\Lambda_{\pi}^{2}+2 \lambda_{\pi}^{2}\right) \bar{\delta}^{2}$ and $\Delta_{\delta} \equiv \frac{1}{2}\left(\Lambda_{\pi}^{2}+2 \lambda_{\pi}^{2}\right) \bar{\sigma}^{2}+\frac{3}{2} \Lambda_{\pi}^{2} \bar{\delta}^{2}$. Therefore, in the chiral limit $m_{u}=m_{d}=0$, see Eq. (4.7), the Hessian matrix of the scalar fields $\left(h_{X}, \sigma, \vec{\delta}\right)$ turns out to be diagonal, with squared masses given by

$$
M_{h_{X}}^{2}=\lambda_{X}^{2} F_{X}^{2}, \quad M_{\sigma}^{2}=\frac{1}{2}\left(\lambda_{\pi}^{2} B_{\pi}^{2}-c_{1} F_{X}\right), \quad M_{\delta}^{2}=\frac{1}{2}\left(\lambda_{\pi}^{2} B_{\pi}^{2}+c_{1} F_{X}\right) .
$$

\section{B. Pseudoscalar sector}

From Eqs. (4.2) and (3.3), it comes out that the Hessian matrix (evaluated at the stationary point $S$ ) is already diagonal with respect to the fields $\pi_{1}$ and $\pi_{2}$, with a common value of the squared masses given by

$$
M_{\pi_{1,2}}^{2}=\frac{1}{2}\left(\lambda_{\pi}^{2} B_{\pi}^{2}-c_{1} \sqrt{2} \bar{\alpha}\right)+\frac{1}{2} \Lambda_{\pi}^{2}\left(\bar{\sigma}^{2}+\bar{\delta}^{2}\right)+\lambda_{\pi}^{2} \bar{\delta}^{2}
$$

The Hessian of the remaining pseudoscalar fields $\left(S_{X}, \eta, \pi_{3}\right)$ turns out to be

$$
\mathcal{H}_{(P S)}=\left(\begin{array}{ccc}
\frac{c_{1}}{2 \sqrt{2} \bar{\alpha}}\left(\bar{\sigma}^{2}-\bar{\delta}^{2}\right)+\frac{A}{\bar{\alpha}^{2}} & -\frac{c_{1}}{\sqrt{2}} \bar{\sigma} & \frac{c_{1}}{\sqrt{2}} \bar{\delta} \\
-\frac{c_{1}}{\sqrt{2}} \bar{\sigma} & \frac{1}{2}\left(\lambda_{\pi}^{2} B_{\pi}^{2}+c_{1} \sqrt{2} \bar{\alpha}\right)+\Delta & \lambda_{\pi}^{2} \bar{\sigma} \bar{\delta} \\
\frac{c_{1}}{\sqrt{2}} \bar{\delta} & \lambda_{\pi}^{2} \bar{\delta} \bar{\sigma} & \frac{1}{2}\left(\lambda_{\pi}^{2} B_{\pi}^{2}-c_{1} \sqrt{2} \bar{\alpha}\right)+\Delta
\end{array}\right),
$$

where $\Delta \equiv \frac{1}{2} \Lambda_{\pi}^{2}\left(\bar{\sigma}^{2}+\bar{\delta}^{2}\right)$. Therefore, in the chiral limit $m_{u}=m_{d}=0$, see Eq. (4.7), the Hessian matrix of the pseudoscalar fields $\left(S_{X}, \eta, \vec{\pi}\right)$ turns out to be diagonal, with squared masses given by

$$
M_{S_{X}}^{2}=\frac{2 A}{F_{X}^{2}}, \quad M_{\eta}^{2}=\frac{1}{2}\left(\lambda_{\pi}^{2} B_{\pi}^{2}+c_{1} F_{X}\right), \quad M_{\pi}^{2}=\frac{1}{2}\left(\lambda_{\pi}^{2} B_{\pi}^{2}-c_{1} F_{X}\right) .
$$

Therefore, in the case $N_{f}=2$, the restoration of the $S U(2)_{L} \otimes S U(2)_{R}$ chiral symmetry manifests itself in the appearance, in the mass spectrum of the effective Lagrangian, of two $q \bar{q}$ chiral multiplets $\left(\frac{1}{2}, \frac{1}{2}\right)$, namely,

$$
\begin{aligned}
(\sigma, \vec{\pi}): & : & M_{\sigma}^{2}=M_{\pi}^{2}=\frac{1}{2}\left(\lambda_{\pi}^{2} B_{\pi}^{2}-c_{1} F_{X}\right), \\
(\eta, \vec{\delta}) & : & M_{\eta}^{2}=M_{\delta}^{2}=\frac{1}{2}\left(\lambda_{\pi}^{2} B_{\pi}^{2}+c_{1} F_{X}\right) .
\end{aligned}
$$

Instead, the squared masses of the $q \bar{q}$ mesonic excitations belonging to a same $U(1)$ chiral multiplet, such as $(\sigma, \eta)$ and $(\vec{\pi}, \vec{\delta})$, remain split by the quantity

$$
\Delta M_{U(1)}^{2} \equiv M_{\eta}^{2}-M_{\sigma}^{2}=M_{\delta}^{2}-M_{\pi}^{2}=c_{1} F_{X},
$$


proportional to the $U(1)$ axial condensate. This result is to be contrasted with the corresponding result obtained in the previous section for the $N_{f} \geq 3$ case, see Eq. (3.9), in which all (scalar and pseudoscalar) $q \bar{q}$ mesonic excitations (described by the field $U$ ) turned out to be degenerate, with squared masses $M_{U}^{2}=\frac{1}{2} \lambda_{\pi}^{2} B_{\pi}^{2}$.

We must now make an important remark about the solution (4.7) that we have found. From the results (4.10) and (4.13) we see that this stationary point is a minimum of the potential, provided that $\lambda_{\pi}^{2} B_{\pi}^{2}>c_{1} F_{X}$; otherwise, the Hessian evaluated at the stationary point would not be positive definite, being $\left.\frac{\partial^{2} \tilde{V}}{\partial \sigma^{2}}\right|_{S}=\left.\frac{\partial^{2} \tilde{V}}{\partial \pi_{a}^{2}}\right|_{S}=\frac{1}{2}\left(\lambda_{\pi}^{2} B_{\pi}^{2}-c_{1} F_{X}\right)<0$. Remembering that, for $T_{\rho_{\pi}}<T<T_{U(1)}, \rho_{\pi} \equiv-\frac{1}{2} B_{\pi}^{2}<0$, the condition for the stationary point (4.7) to be a minimum can be written as

$$
\mathcal{G}_{\pi} \equiv c_{1} F_{X}+2 \lambda_{\pi}^{2} \rho_{\pi}=c_{1} F_{X}-\lambda_{\pi}^{2} B_{\pi}^{2}<0, \quad \text { i.e. : } \quad \rho_{\pi}<-\frac{c_{1} F_{X}}{2 \lambda_{\pi}^{2}} .
$$

In other words, assuming $c_{1} F_{X}>0$ and approximately constant (as a function of the temperature $T$ ) around $T_{\rho_{\pi}}$, we have that the stationary point (4.7) is a solution, i.e., a minimum of the potential, not immediately above $T_{\rho_{\pi}}$, where the parameter $\rho_{\pi}$ vanishes (see Table 1) and $\mathcal{G}_{\pi}$ is positive, but (assuming that $\lambda_{\pi}^{2} B_{\pi}^{2}$ becomes large enough increasing $T$, starting from $\lambda_{\pi}^{2} B_{\pi}^{2}=0$ at $T=T_{\rho_{\pi}}$ ) only for temperatures that are sufficiently higher than $T_{\rho_{\pi}}$, so that the condition (4.16) is satisfied, i.e., only for $T>T_{c}$, where $T_{c}$ is defined by the condition $\mathcal{G}_{\pi}\left(T=T_{c}\right)=0$, and it is just what we can call the chiral transition temperature. In fact, for $T>T_{c}$ the solution (4.7) is valid, and the $S U(2)_{L} \otimes S U(2)_{R}$ chiral symmetry is restored. Therefore, differently from the case $N_{f} \geq 3$ discussed in the previous section, where $T_{c} \equiv T_{\rho_{\pi}}$, we have here that $T_{c}>T_{\rho_{\pi}}$.

Now the question is as follows: What happens for $T_{\rho_{\pi}}<T<T_{c}$ ?

\section{Study of the solution for $T_{\rho_{\pi}}<T<T_{c}$}

One immediately sees that, when

$$
\mathcal{G}_{\pi} \equiv c_{1} F_{X}+2 \lambda_{\pi}^{2} \rho_{\pi}=c_{1} F_{X}-\lambda_{\pi}^{2} B_{\pi}^{2} \geq 0
$$

Eqs. (4.5) also admit the solution (in the chiral limit $m_{u}=m_{d}=0$ )

$$
\bar{\sigma}=\frac{1}{\Lambda_{\pi}} \sqrt{c_{1} \sqrt{2} \bar{\alpha}-\lambda_{\pi}^{2} B_{\pi}^{2}} \equiv \sigma_{0}, \quad \bar{\delta}=0,
$$

with $\bar{\alpha}$ defined implicitly by the third equation in Eqs. (4.5), i.e.,

$$
\lambda_{X}^{2}\left(\bar{\alpha}^{2}-\frac{F_{X}^{2}}{2}\right) \bar{\alpha}=\frac{c_{1}}{2 \sqrt{2} \Lambda_{\pi}^{2}}\left(c_{1} \sqrt{2} \bar{\alpha}-\lambda_{\pi}^{2} B_{\pi}^{2}\right) .
$$


This solution, being of the form $\bar{U}=\frac{\sigma_{0}}{\sqrt{2}} \mathbf{I}$, with $\sigma_{0}>0$, spontaneously breaks the chiral symmetry down to the vectorial subgroup $U(2)_{V}$. It is easy to verify that, by virtue of the condition (4.17), Eq. (4.19) admits a unique solution such that $\bar{\alpha} \geq \frac{F_{X}}{\sqrt{2}} \geq \frac{\lambda_{\pi}^{2} B_{\pi}^{2}}{\sqrt{2} c_{1}}$ [where the last inequality comes from the condition (4.17)], thus leading to a well-defined solution (4.18) for $\bar{\sigma}$. When, in particular, $\mathcal{G}_{\pi}=0$ (i.e., when $T=T_{c}$ ), then the solution coincides with Eq. (4.7), being $\bar{\alpha}=\frac{F_{X}}{\sqrt{2}}$ and $\bar{\sigma}=\bar{\delta}=0$. Instead, for $\mathcal{G}_{\pi}>0$ (i.e., for $T<T_{c}$ ), one has that $\bar{\alpha}>\frac{F_{X}}{\sqrt{2}}$ and $\bar{\sigma}>0$. By studying the matrix of the second derivatives of the potential, calculated in this stationary point, one immediately verifies that this solution is a minimum of the potential and that the masses of the pseudoscalar $q \bar{q}$ excitations $\pi_{a}$ (the pions) vanish; i.e., the $\pi_{a}$ are the three Goldstone bosons coming from the breaking of $S U(2)_{L} \otimes S U(2)_{R}$ down to $S U(2)_{V}$. Obviously, the solution (4.18)-(4.19) continues to be valid also for $T<T_{\rho_{\pi}}$, where $\rho_{\pi} \equiv \frac{A_{\pi}^{2}}{2}>0$, provided that one substitutes $B_{\pi}^{2}$ with $-2 \rho_{\pi}=-A_{\pi}^{2}$.

\section{Chiral condensate for $T>T_{c}$ and for $T<T_{c}$}

It is well known that, since the derivative of the QCD Hamiltonian with respect to the quark mass $m_{l}$ is the operator $\bar{q}_{l} q_{l}$ (being $\delta \mathcal{L}_{Q C D}^{(\text {mass })}=-\sum_{l=1}^{N_{f}} m_{l} \bar{q}_{l} q_{l}$ ), then the corresponding derivative of the vacuum energy represents the vacuum expectation value of $\bar{q}_{l} q_{l}$, i.e., the so-called chiral condensate. In terms of the effective Lagrangian, this means

$$
\left\langle\bar{q}_{l} q_{l}\right\rangle=\frac{\partial \bar{V}}{\partial m_{l}}
$$

where $\bar{V}=\tilde{V}\left(\bar{U}, \bar{U}^{\dagger}, \bar{X}, \bar{X}^{\dagger}\right)=V\left(\bar{U}, \bar{U}^{\dagger}, \bar{X}, \bar{X}^{\dagger}\right)$ is the vacuum expectation value of the potential of the effective Lagrangian. Using the fact that $\bar{\beta}=\bar{\eta}=\bar{\pi}_{a}=\bar{\delta}_{1}=\bar{\delta}_{2}=0$, we find, from Eqs. (3.3) and (4.2),

$$
\begin{aligned}
\bar{V}= & \frac{1}{8} \lambda_{\pi}^{2} B_{\pi}^{4}+\frac{1}{8} \Lambda_{\pi}^{2}\left(\bar{\sigma}^{2}+\bar{\delta}^{2}\right)^{2}+\frac{1}{2} \lambda_{\pi}^{2} \bar{\sigma}^{2} \bar{\delta}^{2}+\frac{1}{4} \lambda_{X}^{2}\left(\alpha^{2}-\frac{F_{X}^{2}}{2}\right)^{2} \\
& +\frac{1}{4}\left(\lambda_{\pi}^{2} B_{\pi}^{2}-\sqrt{2} c_{1} \bar{\alpha}\right) \bar{\sigma}^{2}+\frac{1}{4}\left(\lambda_{\pi}^{2} B_{\pi}^{2}+\sqrt{2} c_{1} \bar{\alpha}\right) \bar{\delta}^{2} \\
& -\frac{B_{m}}{2}\left[\left(m_{u}+m_{d}\right) \bar{\sigma}+\left(m_{u}-m_{d}\right) \bar{\delta}\right],
\end{aligned}
$$

which, when inserted into Eq. (4.20), gives

$$
\begin{aligned}
& \left\langle\bar{q}_{u} q_{u}\right\rangle=\frac{\partial \bar{V}}{\partial m_{u}}=-\frac{B_{m}}{2}(\bar{\sigma}+\bar{\delta}), \\
& \left\langle\bar{q}_{d} q_{d}\right\rangle=\frac{\partial \bar{V}}{\partial m_{d}}=-\frac{B_{m}}{2}(\bar{\sigma}-\bar{\delta}),
\end{aligned}
$$


having used Eqs. (4.5) for the vacuum expectation values $\bar{\sigma}, \bar{\delta}$, and $\bar{\alpha}$. Substituting the solutions (4.6) into the expressions (4.22), we find that, for $T>T_{c}$,

$$
\begin{aligned}
\left\langle\bar{q}_{u} q_{u}\right\rangle & \simeq-\frac{B_{m}^{2}}{\lambda_{\pi}^{4} B_{\pi}^{4}-c_{1}^{2} F_{X}^{2}}\left(m_{u} \lambda_{\pi}^{2} B_{\pi}^{2}+m_{d} c_{1} F_{X}\right), \\
\left\langle\bar{q}_{d} q_{d}\right\rangle & \simeq-\frac{B_{m}^{2}}{\lambda_{\pi}^{4} B_{\pi}^{4}-c_{1}^{2} F_{X}^{2}}\left(m_{d} \lambda_{\pi}^{2} B_{\pi}^{2}+m_{u} c_{1} F_{X}\right) .
\end{aligned}
$$

As we see, the chiral condensate vanishes in the chiral limit $m_{u}=m_{d}=0$, signalling the restoring of the chiral symmetry. Concerning the dependence on the quark masses, we observe that, in agreement with what was already found in Ref. [10] for $N_{f} \geq 3$, also for the case $N_{f}=2$ the expression (4.23) for the chiral condensate comes out to be the sum of two contributions, $\left\langle\bar{q}_{l} q_{l}\right\rangle=\mathcal{O}_{1}\left(m_{l}\right)+\mathcal{O}_{2}\left(\prod_{k \neq l} m_{k}\right)$, for which the diagrammatic interpretation is rather simple (see Fig. 1): the first term $\mathcal{O}_{1}\left(m_{l}\right)$ corresponds to a diagram with the insertion of a mass operator $-m_{l} \bar{q}_{l} q_{l}$, while the second term $\mathcal{O}_{2}\left(\prod_{k \neq l} m_{k}\right)$ clearly corresponds to a diagram with the insertion of the $2 N_{f}$-quark effective vertex (" $\gamma$ ") associated with the $U(1)$ axial condensate $\bar{X}$.

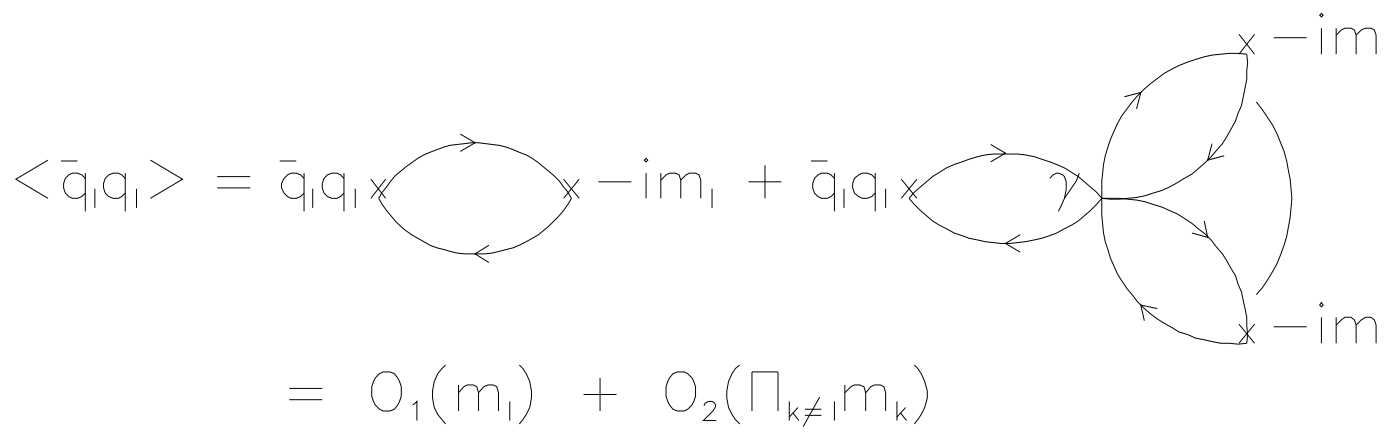

Figure 1: The chiral condensate above $T_{c}$.

Instead, for $T<T_{c}$, one finds, using the solution (4.18) (with the substitution $B_{\pi}^{2} \rightarrow$ $-2 \rho_{\pi} \equiv-A_{\pi}^{2}$, if it is also $\left.T<T_{\rho_{\pi}}\right)$, that, in the chiral limit $m_{u}=m_{d}=0$,

$$
\left\langle\bar{q}_{u} q_{u}\right\rangle=\left\langle\bar{q}_{d} q_{d}\right\rangle=-\frac{1}{2} B_{m} \sigma_{0} \equiv-\frac{1}{2} B_{m} F_{\pi}
$$

since, in this case, $\bar{U}=\frac{\sigma_{0}}{\sqrt{2}} \mathbf{I}$, and, therefore, on the basis of what we have observed in Sec. 2 [see, in particular, Eq. (2.9) and the third footnote], $\sigma_{0}$ must be identified with the pion decay constant: $\sigma_{0} \equiv F_{\pi}$. 


\section{Comments on the results and conclusions}

Let us first summarize the results that we have found.

Chiral symmetry restoration at nonzero temperature is often studied in the framework of the effective Lagrangian (2.1)-(2.3) (see, e.g., Refs. [16, 17, 18, 19]), written in terms of the (quark-bilinear) mesonic effective field $U$ as (in the chiral limit $M=0) \mathcal{L}_{1}=\mathcal{L}_{0}+\mathcal{L}_{I}$, where $\mathcal{L}_{0}$ describes a kind of linear sigma model [see Eq. (2.2)], while $\mathcal{L}_{I}$ is an interaction term of the form $\mathcal{L}_{I}=c_{I}\left[\operatorname{det} U+\operatorname{det} U^{\dagger}\right]$. However, as was noticed by Witten [24], Di Vecchia, and Veneziano [25], this type of anomalous term does not correctly reproduce the U(1) axial anomaly of the fundamental theory (i.e., of the QCD), which is instead correctly implemented in the effective Lagrangian $\mathcal{L}_{2}$, written in Eq. (2.8), which was derived in Refs. [24, 25, 26, 27, 28]. For studying the phase structure of the theory at finite temperature, all the parameters appearing in the effective Lagrangian must be considered as functions of the physical temperature $T$. However, the anomalous term in Eq. (2.8) makes sense only in the low-temperature phase $\left(T<T_{c}\right)$, and it is singular for $T>T_{c}$, where the vacuum expectation value of the mesonic field $U$ vanishes. On the contrary, the interaction term $\mathcal{L}_{I}$ behaves well both in the low- and high-temperature phases.

To overcome the above-mentioned problems, we have considered a modified effective Lagrangian (which was originally proposed in Refs. [10, 11, 12] and elaborated on in Refs. [13, 14, 15]), which generalizes the two effective Lagrangians $\mathcal{L}_{1}$ and $\mathcal{L}_{2}$ mentioned above, in such a way that it correctly satisfies the transformation property (2.6) under the chiral group but also includes an interaction term containing the determinant of the mesonic field $U$, of the kind of that in Eq. (2.3), assuming that there is a $U(1)_{A}$-breaking condensate that (possibly) survives across the chiral transition at $T_{c}$, staying different from zero up to a temperature $T_{U(1)}>T_{c}$. The modified effective Lagrangian is written in terms of the $q \bar{q}$ mesonic effective field $U$ and of the $2 N_{f}$-quark (exotic) mesonic field $X$, associated with the $U(1)$ axial condensate, and it is given by Eqs. (2.17)-(2.18). In particular, the potential term $V\left(U, U^{\dagger}, X, X^{\dagger}\right)$, written in Eq. (2.15), contains an interaction term between the $U$ and $X$ fields, i.e., $\mathcal{L}_{i n t}=\frac{c_{1}}{2 \sqrt{2}}\left[X^{\dagger} \operatorname{det} U+X \operatorname{det} U^{\dagger}\right]$, which is very similar to the interaction term $\mathcal{L}_{I}$ that we have discussed above for the effective Lagrangian $\mathcal{L}_{1}$. Even if this term is not anomalous, being invariant under the chiral group $U\left(N_{f}\right)_{L} \otimes U\left(N_{f}\right)_{R}$, by virtue of Eqs. (2.4) and (2.16), nevertheless, if the field $X$ has a (real) nonzero vacuum

expectation value $\bar{X}$ (the $U(1)$ axial condensate), then, writing $X=\left(\bar{X}+h_{X}\right) e^{i \frac{S_{X}}{X}}$ (with 
$\left.\bar{h}_{X}=\bar{S}_{X}=0\right)$ and expanding in powers of the excitations $h_{X}$ and $S_{X}$, one recovers, at the leading order, an interaction term of the form $\mathcal{L}_{I}: \mathcal{L}_{i n t}=c_{I}\left[\operatorname{det} U+\operatorname{det} U^{\dagger}\right]+\ldots$, with $c_{I}=\frac{c_{1} \bar{X}}{2 \sqrt{2}}$. In Secs. 3 and 4 of this paper, we have analyzed in detail the effects of assuming a nonzero value of the $U(1)$ axial condensate $\bar{X}$ on the scalar and pseudoscalar mesonic mass spectrum above the chiral transition temperature $\left(T>T_{c}\right)$, both for the case $N_{f} \geq 3$ (Sec. 3) and for the case $N_{f}=2$ (Sec. 4).

In particular, in the chiral limit $M=0$, one has that, for $T>T_{c}, \bar{U}=0, \bar{X}=\sqrt{\rho_{X}} \equiv$ $\frac{F_{X}}{\sqrt{2}}$ [where $\rho_{X} \equiv \frac{F_{X}^{2}}{2}$, see Eq. (3.1), is the parameter appearing in the potential term (2.15)], which means that the $S U\left(N_{f}\right)_{L} \otimes S U\left(N_{f}\right)_{R}$ chiral symmetry is restored, while the $U(1)$ axial symmetry is broken by the $U(1)$ axial condensate $\bar{X}$. Concerning the mass spectrum of the effective Lagrangian, first of all we have two exotic $2 N_{f}$-quark mesonic excitations, described by the scalar singlet field $h_{X} \sim \operatorname{det}\left(\bar{q}_{s L} q_{t R}\right)+\operatorname{det}\left(\bar{q}_{s R} q_{t L}\right)$ and by the pseudoscalar singlet field $S_{X} \sim i\left[\operatorname{det}\left(\bar{q}_{s L} q_{t R}\right)-\operatorname{det}\left(\bar{q}_{s R} q_{t L}\right)\right]$, with squared masses given by $M_{h_{X}}^{2}=2 \lambda_{X}^{2} \rho_{X}^{2}=\lambda_{X}^{2} F_{X}^{2}$ and $M_{S_{X}}^{2}=\frac{A}{\bar{X}}=\frac{2 A}{F_{X}^{2}}$. In particular, the physical interpretation of the pseudoscalar singlet excitation $S_{X}$ is rather obvious, and it was already discussed in Ref. [10]; it is nothing but the would-be Goldstone particle coming from the breaking of the $U(1)$ axial symmetry. In fact, neglecting the anomaly, it has zero mass in the chiral limit of zero quark masses. Yet, considering the anomaly, it acquires a topological squared mass proportional to the topological susceptibility $A$ of the pure YM theory, as required by the Witten-Veneziano mechanism [5, 6].

In addition, we have the usual $2 N_{f}^{2} q \bar{q}$ mesonic excitations described by the field $U$. In the case $N_{f}=2$, the restoration of the $S U(2)_{L} \otimes S U(2)_{R}$ chiral symmetry manifests itself in the appearance, in the mass spectrum of the effective Lagrangian, of two $q \bar{q}$ chiral multiplets $\left(\frac{1}{2}, \frac{1}{2}\right)$, namely, using for $U$ the parametrization (4.1) in terms of the fields $\sigma$, $\eta, \vec{\delta}$, and $\vec{\pi},(\sigma, \vec{\pi})$, with masses $M_{\sigma}^{2}=M_{\pi}^{2}=\frac{1}{2}\left(\lambda_{\pi}^{2} B_{\pi}^{2}-\sqrt{2} c_{1} \bar{X}\right)$, and $(\eta, \vec{\delta})$, with masses $M_{\eta}^{2}=M_{\delta}^{2}=\frac{1}{2}\left(\lambda_{\pi}^{2} B_{\pi}^{2}+\sqrt{2} c_{1} \bar{X}\right)$. Instead, the squared masses of the $q \bar{q}$ mesonic excitations belonging to a same $U(1)$ chiral multiplet, such as $(\sigma, \eta)$ and $(\vec{\pi}, \vec{\delta})$, remain split by the quantity*

$$
\Delta M_{U(1)}^{2} \equiv M_{\eta}^{2}-M_{\sigma}^{2}=M_{\delta}^{2}-M_{\pi}^{2}=\sqrt{2} c_{1} \bar{X},
$$

proportional to the $U(1)$ axial condensate $\bar{X}=\frac{F_{X}}{\sqrt{2}}$. This result is to be contrasted with

${ }^{*}$ Since, as we have seen in the previous section, $\sqrt{2} c_{1} \bar{X}=c_{1} F_{X} \geq 0$, where we have also included the equality sign to take into account the limit cases in which $c_{1}=0$ and/or $\bar{X}=0$ (see the discussion below), Eq. (5.1) implies that $M_{\pi} \leq M_{\delta}$, which can be proved to be an exact inequality in QCD (see, e.g., Ref. [32] and references therein). 
the corresponding result obtained in Sec. 3 for the $N_{f} \geq 3$ case, see Eq. (3.9), where all (scalar and pseudoscalar) $q \bar{q}$ mesonic excitations (described by the field $U$ ) turned out to be degenerate, with squared masses $M_{U}^{2}=\frac{1}{2} \lambda_{\pi}^{2} B_{\pi}^{2}$. (The result that we have obtained for $N_{f} \geq 3$ is in agreement with the result that was found in Ref. [33], where simple grouptheoretical arguments were used to demonstrate that in the high-temperature chirally restored phase of QCD with $N_{f}$ massless flavors, all $n$-point correlation functions of quark bilinears with $n<N_{f}$ are invariant under $U(1)$ axial transformations; in particular, for $N_{f} \geq 3$, all two-point correlation functions of quark bilinears are invariant under $U(1)$ axial transformations, and, as a consequence, all $q \bar{q}$ mesonic excitations are degenerate.)

This difference in the mass spectrum of the $q \bar{q}$ mesonic excitations (described by the field $U$ ) for $T>T_{c}$ between the case $N_{f}=2$ and the case $N_{f} \geq 3$ is due to the different role of the interaction term $\mathcal{L}_{\text {int }}=c_{I}\left[\operatorname{det} U+\operatorname{det} U^{\dagger}\right]+\ldots$ in the two cases. When $N_{f}=2$, this term is (at the lowest order) quadratic in the fields $U$ so that it contributes to the squared mass matrix. Instead, when $N_{f} \geq 3$, this term is (at the lowest order) an interaction term of order $N_{f}$ in the fields $U$ (e.g., a cubic interaction term for $N_{f}=3$ ) so that, in the chiral limit, when $\bar{U}=0$, it does not affect the masses of the $q \bar{q}$ mesonic excitations.

Alternatively, we can also explain the difference by using a "diagrammatic" approach, i.e., by considering, for example, the diagrams that contribute to the following quantity $\mathcal{D}_{U(1)}$, defined as the difference between the correlators for the $\delta^{+}$and $\pi^{+}$channels:

$$
\begin{aligned}
& \mathcal{D}_{U(1)}(x) \equiv\left\langle T O_{\delta^{+}}(x) O_{\delta^{+}}^{\dagger}(0)\right\rangle-\left\langle T O_{\pi^{+}}(x) O_{\pi^{+}}^{\dagger}(0)\right\rangle \\
& \quad=2\left[\left\langle T \bar{u}_{R} d_{L}(x) \bar{d}_{R} u_{L}(0)\right\rangle+\left\langle T \bar{u}_{L} d_{R}(x) \bar{d}_{L} u_{R}(0)\right\rangle\right] .
\end{aligned}
$$

What happens below and above $T_{c}$ ? For $T<T_{c}$, in the chiral limit $m_{1}=\ldots m_{N_{f}}=0$, the left-handed and right-handed components of a given light quark flavor can be connected through the $q \bar{q}$ chiral condensate, giving rise to a nonzero contribution to the quantity $\mathcal{D}_{U(1)}(x)$ in Eq. (5.2). But for $T>T_{c}$, the $q \bar{q}$ chiral condensate is zero, and, therefore, also the quantity $\mathcal{D}_{U(1)}(x)$ should be zero for $T>T_{c}$, unless there is a nonzero $U(1)$ axial condensate $\bar{X}$; in that case, one should also consider the diagram with the insertion of the $2 N_{f}$-quark effective vertex ( $\gamma$ : see Fig. 1 in Sec. 4) associated with the $U(1)$ axial condensate $\bar{X}$. For $N_{f}=2$, all the left-handed and right-handed components of the up and down quark fields in Eq. (5.2) can be connected through the four-quark effective vertex $\gamma$, giving rise to a nonzero contribution to the quantity $\mathcal{D}_{U(1)}(x)$. Instead, for $N_{f}=3$ the six-quark effective vertex $\gamma$ also generates a couple of right-handed and left-handed 
strange quarks, which, for $T>T_{c}$, can only be connected through the mass operator $-m_{s} \bar{q}_{s} q_{s}$, so that (differently from the case $N_{f}=2$ ) this contribution to the quantity $\mathcal{D}_{U(1)}(x)$ should vanish in the chiral limit; this implies that, for $N_{f}=3$ and $T>T_{c}$, the $\vec{\delta}$ and $\vec{\pi}$ correlators are identical, and, as a consequence, also $M_{\delta}=M_{\pi}$. This argument can be easily generalized to include also the other meson channels and to the case $N_{f}>3$.

Finally, let us see how our results for the mass spectrum compare with the available lattice results.

As we have already said in the introduction, information on the mass spectrum of the $q \bar{q}$ mesonic excitations of the theory can be obtained by studying the two-point correlation functions of proper quark-bilinear operators: lattice results for the case $N_{f}=2$ already exist in the literature, even if the situation is, at the moment, a bit controversial. In fact, there are lattice results [34, 35, 36, 37, 38, 39, 40, 41, some of them obtained using the socalled staggered fermions on the lattice and some others using the so-called domain-wall fermions on the lattice, which indicate the nonrestoration of the $U(1)$ axial symmetry above the chiral transition at $T_{c}$, in the form of a small (but nonzero) splitting between the $\vec{\delta}$ and $\vec{\pi}$ correlators above $T_{c}$, up to $\sim 1.2 T_{c} \oplus$ In terms of our result (5.1), we would interpret this by saying that, for $T>T_{c}$, there is still a nonzero $U(1)$ axial condensate, $\bar{X}>0$, so that $c_{I}=\frac{c_{1} \bar{X}}{2 \sqrt{2}}>0$ and the above-mentioned interaction term, containing the determinant of the mesonic field $U$, is still effective for $T>T_{c}$.

However, recently, other lattice results, obtained using the so-called overlap fermions on the lattice, have been reported [42], which do not show evidence of the above-mentioned splitting above $T_{c}$, so indicating an effective restoration of the $U(1)$ axial symmetry above $T_{c}$, at least, at the level of the $q \bar{q}$ mesonic mass spectrum (see also Ref. [43], where the same conclusions have been derived analytically but always using the overlap lattice fermions, with the help of certain assumptions). In terms of our result (15.1), we would interpret this by saying that, for $T>T_{c}$, one has $c_{1} \bar{X}=0$, so that $c_{I}=\frac{c_{1} \bar{X}}{2 \sqrt{2}}=0$ and

\footnotetext{
${ }^{\dagger}$ We must point out that some of the above-mentioned lattice results [39, 40, 41] refer, properly speaking, neither to the case $N_{f}=2$ nor to the case $N_{f}=3$ but to the (more realistic) case " $N_{f}=2+1$," in which there are two (up and down) very light (eventually massless) quark flavors and one massive strange quark with a realistic mass $m_{s} \sim 100 \mathrm{MeV}$. However, it is commonly believed (see, e.g., Refs. [19] and references therein) that, due to the large mass of the strange quark, this case, at least in the vicinity of the phase transition at $T_{c}$, is closer to the ideal case $N_{f}=2$ (obtained in the limit $m_{s} \rightarrow \infty$ ) rather than to the ideal case $N_{f}=3$ (obtained in the limit $m_{s} \rightarrow 0$ ). Moreover, the fact that also, in this case, a splitting is observed between the $\vec{\delta}$ and $\vec{\pi}$ correlators immediately above $T_{c}$ can be considered (on the basis of our previous arguments) as an a posteriori confirmation of this expectation.
} 
the above-mentioned interaction term, containing the determinant of the mesonic field $U$, is not present for $T>T_{c}$. For example, it could be that also the $U(1)$ axial condensate $\bar{X}$ (like the usual chiral condensate $\langle\bar{q} q\rangle$ ) vanishes at $T=T_{c}$, i.e., using the notation introduced in Sec. 2 (see Table 1), that $T_{U(1)}=T_{c}$. (Or, even more drastically, it could be that, at least for $N_{f}=2$, there is simply no genuine $U(1)$ axial condensate ....) In this case, in order to preserve the consistency of our effective model, we should require that also the pure-gauge topological susceptibility $A(T)$ vanishes immediately above the critical temperature $T_{c}$; otherwise, the anomalous term in Eq. (2.18) would be singular above the critical temperature $T_{c}$, where the vacuum expectation values of the mesonic fields vanish (in the chiral limit $M=0$ ). However, lattice results show that the pure-gauge topological susceptibility $A(T)$ is approximately constant up to the critical temperature $T_{c}$, and then it has a sharp decrease above the transition, but it remains different from zero, at least up to $\sim 1.2 T_{c}$ (this suppression for $T>T_{c}$, however, increases when increasing the number $N_{c}$ of colors, thus hinting at a vanishing large- $N_{c}$ limit of $A(T)$ for $T>T_{c}$, as it was suggested in Ref. [44]. See Ref. [45] for a recent review on these problems.) We recall that, in the Witten-Veneziano mechanism [5, 6], a (no matter how small) value different from zero for $A$ is related to the breaking of the $U(1)$ axial symmetry, since it implies the existence of a pseudoscalar and flavor-singlet would-be Goldstone particle; thus, a (small) nonzero value of $A(T)$ for $T>T_{c}$ should imply a (presumably small) nonzero value of the $U(1)$ axial condensate $\bar{X}$.

Alternatively, one could of course explain the (possible) vanishing of the coefficient $c_{I}=\frac{c_{1} \bar{X}}{2 \sqrt{2}}$ of the interaction term containing the determinant of the mesonic field $U$ above $T_{c}$ simply by assuming that the coefficient $c_{1}$ (possibly) vanishes above $T_{c}$. (The possibility that $c_{1} \equiv 0$ at every temperature $T$, including $T=0$, must be discarded if we also assume that there is a genuine nonzero $U(1)$ axial condensate $\bar{X}$, since, as it was shown in Appendix B of Ref. [15], this hypothesis would lead to wrong predictions for the pseudoscalar-meson mass spectrum at $T=0$.)

In conclusion, further work will be necessary, both from the analytical point of view but especially from the numerical point of view (i.e., by lattice calculations), in order to unveil the persistent mystery of the fate of the $U(1)$ axial symmetry at finite temperature. 


\section{Acknowledgments}

A. Mordà has been supported by the OCEVU Labex (Grant No. ANR-11-LABX-0060) and by the A*MIDEX project (Project No. ANR-11-IDEX-0001-02), funded by the "Investissements d'Avenir" French government program managed by the ANR. 


\section{References}

[1] S. Weinberg, The Quantum Theory of Fields, Vol.2: Modern Applications (Cambridge University Press, Cambridge, UK, 1995), Chap. 19.

[2] F. Karsch, Lect. Notes Phys. 583, 209 (2002);

A. Bazavov et al. (HotQCD Collaboration), Phys. Rev. D 85, 054503 (2012).

[3] S. Weinberg, Phys. Rev. D 11, 3583 (1975).

[4] G. 't Hooft, Phys. Rev. Lett. 37, 8 (1976);

G. 't Hooft, Phys. Rev. D 14, 3432 (1976) [Erratum-ibid. 18, 2199 (1978)].

[5] E. Witten, Nucl. Phys. B 156, 269 (1979).

[6] G. Veneziano, Nucl. Phys. B 159, 213 (1979).

[7] D.J. Gross, R.D. Pisarski, and L.G. Yaffe, Rev. Mod. Phys. 53, 43 (1981).

[8] C. DeTar and J. Kogut, Phys. Rev. Lett. 59, 399 (1987);

C. DeTar and J. Kogut, Phys. Rev. D 36, 2828 (1987).

[9] E. Shuryak, Comments Nucl. Part. Phys. 21, 235 (1994).

[10] E. Meggiolaro, Z. Phys. C 62, 669 (1994).

[11] E. Meggiolaro, Z. Phys. C 62, 679 (1994).

[12] E. Meggiolaro, Z. Phys. C 64, 323 (1994).

[13] M. Marchi and E. Meggiolaro, Nucl. Phys. B 665, 425 (2003).

[14] E. Meggiolaro, Phys. Rev. D 69, 074017 (2004).

[15] E. Meggiolaro, Phys. Rev. D 83, 074007 (2011).

[16] R.D. Pisarski and F. Wilczek, Phys. Rev. D 29, 338 (1984).

[17] J.T. Lenaghan, D.H. Rischke, and J. Schaffner-Bielich, Phys. Rev. D 62, 085008 (2000).

[18] D. Röder, J. Ruppert, and D. Rischke, Phys Rev. D 68, 016003 (2003). 
[19] A. Butti, A. Pelissetto, and E. Vicari, J. High Energy Phys. 08 (2003) 029;

F. Basile, A. Pelissetto, and E. Vicari, Proc. Sci. LAT2005 (2005) 199;

A. Pelissetto and E. Vicari, arXiv:1309.5446 [Phys. Rev. D (to be published)].

[20] M. Levy, Nuovo Cimento A 52, 23 (1967).

[21] W.A. Bardeen and B.W. Lee, Phys. Rev. 177, 2389 (1969).

[22] S. Gasiorowicz and D.A. Geffen, Rev. Mod. Phys. 41, 531 (1969).

[23] G. 't Hooft, Phys. Rep. 142, 357 (1986).

[24] E. Witten, Ann. Phys. (N.Y.) 128, 363 (1980).

[25] P. Di Vecchia and G. Veneziano, Nucl. Phys. B 171, 253 (1980).

[26] C. Rosenzweig, J. Schechter, and C.G. Trahern, Phys. Rev. D 21, 3388 (1980).

[27] K. Kawarabayashi and N. Ohta, Nucl. Phys. B 175, 477 (1980).

[28] P. Nath and R. Arnowitt, Phys. Rev. D 23, 473 (1981).

[29] M. Kobayashi and T. Maskawa, Prog. Theor. Phys. 44, 1422 (1970).

[30] T. Kunihiro, Prog. Theor. Phys. 122, 255 (2009).

[31] A. Di Giacomo and E. Meggiolaro, Nucl. Phys. B, Proc. Suppl. 42, 478 (1995).

[32] A. Smilga, Lectures on Quantum Chromodynamics (World Scientific, Singapore, 2001), Lecture 14.

[33] M.C. Birse, T.D. Cohen, and J.A. McGovern, Phys. Lett. B 388, 137 (1996).

[34] C. Bernard et al., Nucl. Phys. B, Proc. Suppl. 53, 442 (1997);

C. Bernard, T. Blum, C. DeTar, S. Gottlieb, U. Heller, J. Hetrick, K. Rummukainen, R. Sugar, D. Toussaint, and M. Wingate, Phys. Rev. Lett. 78, 598 (1997).

[35] J.B. Kogut, J.-F. Lagaë, and D.K. Sinclair, Phys. Rev. D 58, 054504 (1998).

[36] S. Chandrasekharan, D. Chen, N.H. Christ, W.-J. Lee, R. Mawhinney, and P.M. Vranas, Phys. Rev. Lett. 82, 2463 (1999). 
[37] F. Karsch, Nucl. Phys. B, Proc. Suppl. 83-84, 14 (2000).

[38] P.M. Vranas, Nucl. Phys. B, Proc. Suppl. 83-84, 414 (2000).

[39] M. Cheng et al., Eur. Phys. J. C 71, 1564 (2011).

[40] A. Bazavov et al. (HotQCD Collaboration), Phys. Rev. D 86, 094503 (2012).

[41] M.I. Buchoff et al. (LLNL/RBC Collaboration), arXiv:1309.4149 [Phys. Rev. D (to be published)].

[42] G. Cossu, S. Aoki, H. Fukaya, S. Hashimoto, T. Kaneko, H. Matsufuru, and J.-I. Noaki, Phys. Rev. D 87, 114514 (2013).

[43] S. Aoki, H. Fukaya, and Y. Taniguchi, Phys. Rev. D 86, 114512 (2012).

[44] D. Kharzeev, R.D. Pisarski, and M.H.G. Tytgat, Phys. Rev. Lett. 81, 512 (1998).

[45] E. Vicari and H. Panagopoulos, Phys. Rep. 470, 93 (2009). 